\title{
La réflectivité de neutrons
}

\author{
C. Fermon
}

DSM/DRECAM/SPEC, CEA/Saclay, 91191 Gif-sur-Yvette, France

\section{Introduction}

La réflectivité de neutrons est une technique de mesure relativement récente. Une présentation générale des méthodes utilisées pour traiter les données est faite ici.

Le contenu de ce cours dépasse largement ce qui sera exposé au cours de l'école mais il vous permettra de voir les différentes approches dans le traitement des données de réflectivité neutronique ou de rayons $X$.

La première partie a pour but de donner les idées générales de la réflectivité avec quelques exemples pédagogiques. Dans une deuxième partie, je ferai une approche de la réflectivité très proche de la réflectivité de la lumière. C'est ce formalisme qui est actuellement principalement utilisé pour les rayons $\mathrm{X}$ et les neutrons non polarisés.

Dans une troisième partie, je vais introduire le spin du neutron et l'utilisation de neutrons polarisés. Dans ce cas, il faut remonter à l'équation de Schrödinger pour résoudre le problème.

J'aborderai dans une quatrième partie, le traitement des données expérimentales et les problèmes liés à la rugosité

Je décrirai ensuite brièvement les appareils puis je finirai par un certain nombre d'exemples illustrant la technique.

\section{Neutron dans le vide}

Nous allons ici résoudre l'équation de Schrödinger pour un neutron se propageant dans le vide en présence d'un champ magnétique. Les résultats nous serviront plus tard et cela permet de manipuler la fonction d'onde du neutron.

Dans ce cas, l'interaction est simplement l'interaction Zeeman avec le champ extérieur

$$
V_{z}(\mathbf{r})=-g_{n} \mu_{n} \sigma . \mathbf{H}_{0}
$$

L'équation de Schrödinger s'écrit donc en prenant l'axe z suivant le champ magnétique.

$$
\frac{\hbar^{2}}{2 m} \Delta \psi+\left[E+g_{n} \mu_{n} \sigma_{z} H_{0}\right] \psi=0
$$

On peut alors écrire, comme le potentiel ne dépend que de $z$, la fonction d'onde sous la forme. 


$$
\psi=e^{i\left(k_{x} x+k_{y} y\right)}\left(\psi_{z}^{+}|+\rangle+\psi_{z}^{-}|-\rangle\right)
$$

L'équation (12) donne deux équations séparées, une pour chaque composante du spineur:

$$
\frac{\hbar^{2}}{2 m} \Delta \psi_{z}^{ \pm}+\left[E-\frac{\hbar^{2}\left(k_{x}^{2}+k_{y}^{2}\right)}{2 m} \pm g_{n} \mu_{n} H_{0}\right] \psi_{z}^{ \pm}=0
$$

Le résultat est :

$$
\begin{aligned}
& \psi_{z}^{ \pm}=e^{i q_{ \pm} z} \\
& \text { avec } q_{ \pm}=\left[\frac{2 m}{\hbar^{2}} E-\left(k_{x}^{2}+k_{y}^{2}\right) \pm \frac{2 m}{\hbar^{2}} g_{n} \mu_{n} H_{0}\right]^{1 / 2}
\end{aligned}
$$

On remarque le passage de $\mathrm{k}$ à $\mathrm{q}$ pour la composante $\mathrm{z}$, qui sera dans la suite la perpendiculaire à la surface. Cela permet de garder la compatibilité avec de nombreux articles et évite de traîner un indice $\mathrm{z}$ tout le temps.

Physiquement, quel est le comportement d'un neutron se propageant dans un champ magnétique ? Le neutron dans un état up, se propagera plus vite que le neutron dans un état down. Cette différence peut pour des champs assez forts se mesurer.

Dans le cas où le neutron n'est pas dans un état propre, mais par exemple quantifié le long de $x$, sa fonction d'onde à $t=0$ est $\frac{1}{\sqrt{2}}(|+\rangle+|-\rangle)$. On décrira alors le neutron comme un objet ayant deux parties se propageant à des vitesses différentes.

Une description plus classique est de considérer le neutron comme ayant une fonction d'onde dépendant du temps. A ce moment, il faut résoudre l'équation de Schrödinger dépendant du temps. On arrive à une solution qui apparaît au premier abord différente : le neutron est un paquet se propageant avec une énergie $E$ et son spin précesse à la fréquence de Larmor autour du champ magnétique. En fait, en introduisant la vitesse du neutron qui relie position et temps, on a vite fait de montrer que la fonction d'onde finale est identique pour des champs extérieur suffisamment faibles.

\subsection{Réflectivité sans magnétisme}

Dans toute cette partie, on oublie le spin du neutron et on va faire réfléchir les neutrons sur des systèmes non magnétiques. On va introduire la notion d'indices. C'est une approche rayons $\mathrm{X}$ et matière molle du formalisme de la réflectivité. Elle est inapplicable pour le magnétisme mais elle permet de voir les choses et de traiter plus simplement la rugosité.

\subsection{Réflexion sur une surface plane, introduction des grandeurs}

Soit un faisceau de neutrons se réfléchissant sur une surface plane avec un angle incident $\theta$. Cette surface (voir Figure 1) est définie par l'interface air ( $n=1)$ - milieu matériel d'indice de réfraction constant $n$. 


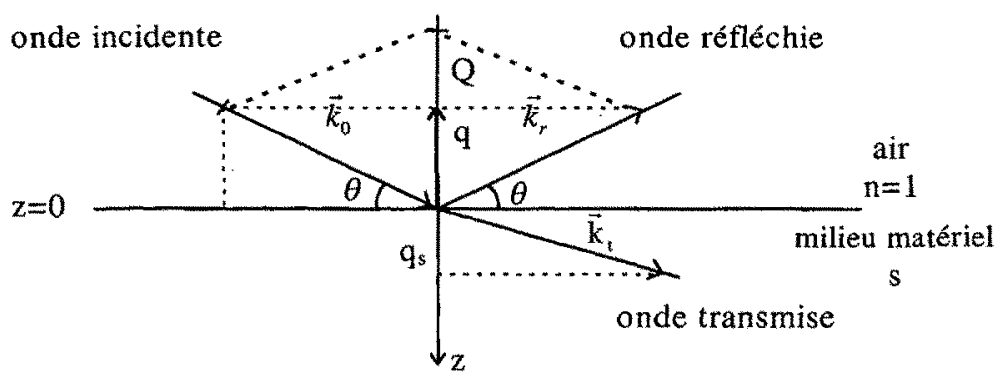

Figure1: Réflexion d'une onde plane sur une surface parfaite.

L'indice n donné sur la figure sera tiré, dans la suite, de l'équation de Schrödinger.

On suppose le milieu (et donc le potentiel $V$ ) invariant selon $x$ et y (le plan xy est parallèle à la surface). On définit alors la projection du vecteur d'onde sur l'axe $\mathrm{z}$, perpendiculaire à la surface, par la variable $Q$ :

$$
\mathrm{Q}=\frac{4 \pi}{\lambda} \sin \theta
$$

$\mathrm{Q}$, le vecteur de diffusion, est la bonne variable pour la réflectivité comme pour la diffraction de neutrons. Souvent on peut rencontrer des gens utilisant $q=Q / 2$

En séparant les variables, $x, y$ et $z$ l'équation de Schrödinger (voir cours d'introduction) peut être réduite sous la forme en absence de champ magnétique extérieur à:

$$
\frac{\hbar^{2}}{2 \mathrm{~m}} \frac{\mathrm{d}^{2} \psi_{z}}{\mathrm{dz}}+\left[\mathrm{E}_{\mathrm{z}}-\mathrm{V}_{\mathrm{z}}\right] \psi_{\mathrm{z}}=0
$$

En dehors du milieu $(z=0, V=0)$, le vecteur de diffusion permet de définir l'énergie $E$ :

Le potentiel $\mathrm{V}_{\mathrm{z}}$ moyen dans la couche s'obtient par intégration du pseudo-potentiel de Fermi :

$$
V_{z}=\frac{1}{v} \int_{v} V(\mathbf{r}) d \mathbf{r}=\frac{2 \pi \hbar^{2}}{m} b \rho
$$

$\rho$ désigne le nombre d'atomes par unité de volume. L'unité usuellement utilisée est le $\mathrm{cm}$.

Les solutions générales de l'équation ci-dessus sont données par :

avec

$$
\psi_{z}=A e^{i q, z}+B e^{-i q, z}
$$

$$
\frac{\hbar^{2}}{2 \mathrm{~m}} q_{s}^{2}=\frac{\hbar^{2}}{2 \mathrm{~m}}\left(q_{0}^{2}-4 \pi b \rho\right)=\mathrm{E}_{z}-\mathrm{V}_{z}
$$


où $\mathrm{q}_{\mathrm{s}}$ est le vecteur de diffusion dans le milieu $\mathrm{s}$.

Les équations de Maxwell permettent d'écrire, pour un champ électrique $E$, une équation de forme équivalente à l'équation(19). 1,2 $^{1,2}$

La continuité des composantes parallèles permet d'écrire

$$
k_{f}^{2}=k_{0}^{2}-4 \pi b \rho
$$

Pour tout matériau homogène et non absorbant, $b$ est une constante réelle et on définit l'indice de réfraction $n$ comme le rapport des vecteurs d'onde dans le matériau et le vide ${ }^{3}$ :

$$
\mathrm{n}=\frac{\mathrm{k}_{\mathrm{t}}}{\mathrm{k}}=1-\frac{\lambda^{2}}{2 \pi} \rho b
$$

La relation de Descartes pour les deux milieux d'indices $n=1$ (air) et $n$ s'écrit :

$$
\cos \theta=n \cos \theta_{s}
$$

Il y a réflexion totale tant que $\theta \leq \theta_{c}$, où $\theta_{c}$ est défini tel que $\theta_{\mathrm{n}}=0$, c'est à dire :

$$
\cos \theta_{\mathrm{c}}=\mathrm{n}
$$

$\mathrm{n}$ étant très proche de 1 on peut écrire :

$$
n^{2} \approx 1-\frac{\lambda^{2}}{\pi} \rho b
$$

D'après (15) et (16), on a :

$$
1-\left(\sin \theta_{r}\right)^{2}=1-\frac{\lambda^{2}}{\pi} \rho b
$$

ce qui équivaut à :

$$
\sin \theta_{\mathrm{c}}=\sqrt{\frac{\rho b}{\pi}} \lambda=\alpha \lambda
$$

où le paramètre $\alpha$ est utilisé pour la caractérisation des miroirs de neutrons.

Ceci permet de définir le paramètre de coupure $\mathrm{q}_{\mathrm{c}}$ tel que :

$$
\mathrm{q}_{\mathrm{c}}=\mathrm{Q}_{\mathrm{c}} / 2=\frac{2 \pi \sin \theta_{\mathrm{c}}}{\lambda}=2 \sqrt{\pi \rho b}
$$




\subsection{Réflexion sur un milieu stratifié}

Soit, d'une manière générale, la propagation d'une onde plane associée aux neutrons dans un milieu homogène $\alpha$ puis un autre milieu homogène $\alpha+1$ à la cote $z_{\alpha}$ La solution de l'équation (18) dans les milieux $\alpha$ et $\alpha+1$ est de la forme :

$$
\begin{aligned}
& \psi_{\alpha}(z)=\mathrm{A}_{\alpha} \exp \left(\mathrm{iq}_{\alpha} \mathrm{z}\right)+\mathrm{B}_{\alpha} \exp \left(-\mathrm{iq}_{\alpha} \mathrm{z}\right) \\
& \psi_{\alpha+1}(z)=\mathrm{A}_{\alpha+1} \exp \left(\mathrm{iq}_{\alpha+1} \mathrm{z}\right)+\mathrm{B}_{\alpha+1} \exp \left(-\mathrm{iq}_{\alpha+1} \mathrm{z}\right)
\end{aligned}
$$

où $\mathrm{i}$ est tel que $\mathrm{i}^{2}=-1$, et $\mathrm{A}_{\alpha}$ et $\mathrm{B}_{\alpha}$ sont, dans le milieu $\alpha$, les amplitudes respectives des faisceaux entrant et sortant, avec :

$$
\begin{aligned}
& \mathrm{q}_{\alpha}^{2}=\mathrm{q}_{0}^{2}-4 \pi \rho b_{\alpha} \\
& \mathrm{q}_{\alpha+1}^{2}=\mathrm{q}_{0}^{2}-4 \pi \rho b_{\alpha+1}
\end{aligned}
$$

Les conditions de continuité à l'interface entre les deux milieux permettent d'écrire :

$$
\left.\begin{array}{l}
\psi_{\alpha \alpha}\left(\mathrm{z}_{\alpha}\right)=\psi_{\alpha+1}\left(\mathrm{z}_{\alpha}\right) \\
\psi_{\alpha}^{\prime}\left(\mathrm{z}_{\alpha}\right)=\psi^{\prime}{ }_{\alpha+1}\left(\mathrm{z}_{\alpha}\right)
\end{array}\right\}
$$

\subsection{Calcul de la réflectivité}

\subsubsection{Interface parfaite : réflectivité de Fresnel}

Les milieux $\alpha$ et $\alpha+1$ sont maintenant remplacés respectivement par l'air et par un substrat :

Soit l'interface sans rugosité entre l'air d'indice 1 et le milieu infini s (substrat) d'indice $n$. La valeur de la réflectivité pour un tel système est appelée réflectivité de Fresnel $\left(R_{F}\right)$.

Dans le substrat, le facteur $\mathrm{B}_{\alpha+l}=\mathrm{B}_{\mathrm{s}}$ de l'equation (20) est nul car aucune partie du faisceau ne provient de $z=\infty$. Dans le milieu s, cette équation s'écrit :

$$
\begin{aligned}
& \psi_{s}(\mathrm{z})=\mathrm{A}_{s} \exp \left(\mathrm{iq}_{s} \mathrm{z}\right) \\
& \psi_{s}^{\prime}(\mathrm{z})=\mathrm{iA_{s }} \mathrm{q}_{s} \exp \left(\mathrm{iq}_{s} \mathrm{z}\right)
\end{aligned}
$$

et l'équation (22) de contínuité à l'interface $(\mathrm{z}=0)$ devient :

$$
A_{0}+B_{0}=A_{S} \text { et } q_{0}\left(A_{0}-B_{0}\right)=A_{s} q_{s}
$$


d'où le calcul à l'interface de $\mathrm{A}$ et $\mathrm{B}$ (amplitudes dans l'air ; on notera sans indices les grandeurs dans l'air) :

$$
\left.\begin{array}{l}
A_{0}=\frac{q_{0}+q_{s}}{2 q_{0}} A_{s} \\
B_{0}=\frac{q_{0}-q_{s}}{2 q_{0}} A_{s}
\end{array}\right\}
$$

Pour calculer la réflectivité on fixe $A_{0}=1, B_{0}=r, A_{s}=t$.

La réflectivité mesurée est donnée par: $R=r^{2}=\left|\frac{B_{0}}{A_{0}}\right|^{2}$

La réflectivité de Fresnel est donc:

$$
R_{F}=\left|\frac{B_{0}}{A_{0}}\right|^{2}=\left|\frac{q_{0}-q_{s}}{q_{0}+q_{s}}\right|^{2}
$$

ou encore :

$$
\mathrm{R}_{\mathrm{F}}=\left|\frac{1-\left(1-\left(\frac{\mathrm{q}_{\mathrm{c}}}{\mathrm{q}_{0}}\right)^{2}\right)^{1 / 2}}{1+\left(1-\left(\frac{\mathrm{q}_{\mathrm{c}}}{\mathrm{q}_{0}}\right)^{2}\right)^{1 / 2}}\right|^{2}
$$

où $q_{c}$ et $q_{s}$ sont définis à partir des équations (19) et (21):

$$
\begin{aligned}
& \mathrm{q}_{\mathrm{c}}^{2}=4 \pi \rho b_{\mathrm{s}} \\
& \mathrm{q}_{\mathrm{s}}^{2}=\mathrm{q}_{\mathrm{o}}^{2}-4 \pi \rho b_{\mathrm{s}}=\mathrm{q}_{\mathrm{o}}^{2}-\mathrm{q}_{\mathrm{c}}^{2}
\end{aligned}
$$

La figure 2 montre l'allure de la courbe $R_{F}\left(q_{0}\right)$ calculée pour le système à une interface : on y voit le plateau de réflexion totale tant que $q_{0}<q_{c}$ et la coupure en $q=q_{c}$. 


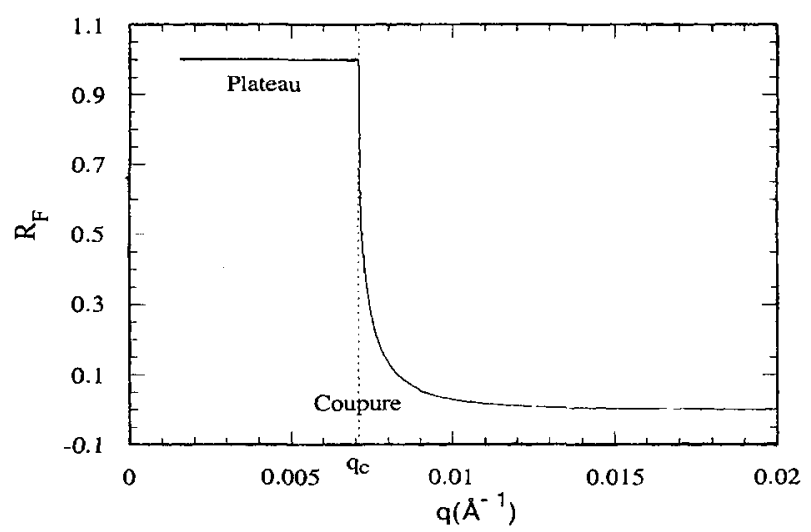

Figure 2 : Représentation de l'équation (27) en fonction de $q_{0}=Q / 2$. Réflectivité de Fresnel sur une surface parfaitement plane.

La fonction est calculée avec $\rho b_{s}=4 \times 10^{-6} \AA^{-2}$ soit $q_{c}=7,08 \times 10^{-3} \AA^{-1}$.

\subsubsection{Couche homogène sur un substrat infini}

Le système considéré est représenté sur la figure 3 . Les trois milieux sont : l'air d'indice $\mathrm{n}=1$ et indexée 0 , une couche homogène d'indice $n_{1}$, d'épaisseur $d$ et de densité de longueur de diffusion cohérente $\rho b_{1}$ indexée1, et le substrat d'indice $n_{s}$, de densité de longueur de diffusion cohérente $\rho b_{s}$ et d'épaisseur infinie. L'interface air-couche est à la cote $\mathrm{z}=0$, l'interface couche-substrat est à $\mathrm{z}=\mathrm{d}$.

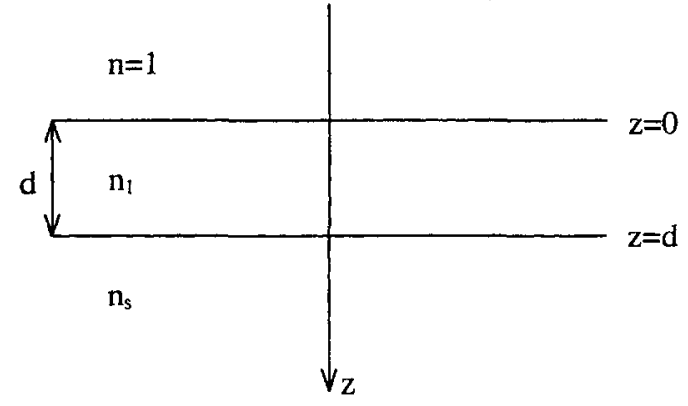

Figure 3: Système à une couche homogène d'épaisseur d déposée sur un substrat s.

Les conditions de continuité (équation (22)) appliquées aux interfaces $\mathrm{z}=0$ et $\mathrm{z}=\mathrm{d}$ permettent d'écrire

$$
\begin{aligned}
& A_{1}+B_{1}=A_{0}+B_{0} \\
& A_{1} q_{1}-B_{1} q_{1}=A_{0} q_{0}-B_{0} q_{0} \\
& A_{1} \exp \left(i q_{1} d\right)+B_{1} \exp \left(-i q_{1} z\right)=A_{s} \exp \left(i q_{s} d\right) \\
& A_{1} q_{1} \exp \left(i q_{l} d\right)-B_{1} q_{1} \exp \left(-i q_{1} z\right)=A_{s} q_{s} \exp \left(i q_{s} d\right)
\end{aligned}
$$


Cette expression montre que le calcul de la réflectivité se prête au formalisme matriciel dans lequel une matrice est écrite pour chaque couche. L'expression précédente est en effet équivalente à :

$$
\left(\begin{array}{l}
A_{0} \\
B_{0}
\end{array}\right)=\left(\begin{array}{ll}
\left(1+q_{1} / q_{0}\right) / 2 & \left(1-q_{1} / q_{0}\right) / 2 \\
\left(1-q_{1} / q_{0}\right) / 2 & \left(1+q_{1} / q_{0}\right) / 2
\end{array}\right)\left(\begin{array}{l}
A_{1} \\
B_{1}
\end{array}\right)
$$

$$
\left(\begin{array}{l}
A_{1} \\
B_{1}
\end{array}\right)=\left(\begin{array}{cc}
\left(1+q_{s} / q_{1}\right) \mathrm{e}^{i q_{s} d} e^{-i q_{1} d} / 2 & \left(1-q_{s} / q_{1}\right) e^{-i q_{s} d} e^{-i q_{1} d} / 2 \\
\left(1-q_{s} / q_{1}\right) e^{i q_{s} d} e^{i q_{1} d} / 2 & \left(1+q_{s} / q_{1}\right) e^{-i q_{s} d} e^{i q_{1} d} / 2
\end{array}\right)\left(\begin{array}{l}
A_{s} \\
B_{S}
\end{array}\right)
$$

avec $\mathrm{q}_{\mathrm{s}}$ et $\mathrm{q}_{1}$ définis par la relation (21):

$$
\begin{aligned}
& q_{\mathrm{s}}^{2}=q_{0}^{2}-4 \pi \rho b_{\mathrm{s}} \\
& q_{1}^{2}=q_{0}^{2}-4 \pi \rho b_{1}
\end{aligned}
$$

De même que précédemment, on fixe $A_{0}=1, B_{0}=r, A_{s}=t$ et $B_{S}=0$.

On en tire $r$ et donc $\mathbf{R}$.

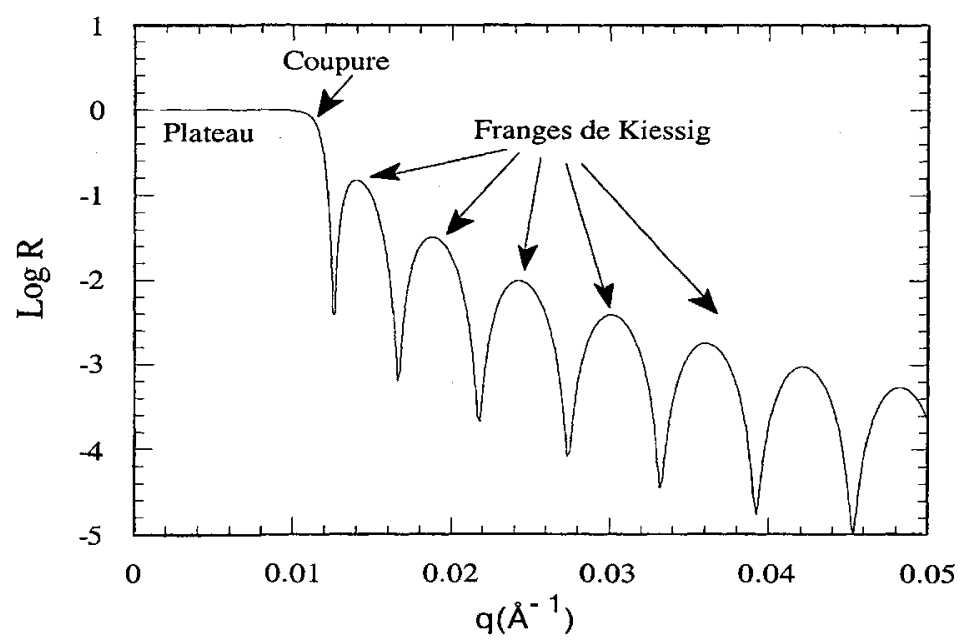

Figure 4 : Représentation du logarithme de la fonction de réflectivité $R$ en fonction de $q$ dans le cas d'une couche homogène déposée sur un substrat. Les valeurs numériques utilisées pour le calcul sont : substrat de silicium : $\rho b_{s}=2 \times 10^{-6} \AA^{-2} ;$ couche de nickel : $\rho b_{1}=9,4 \times 10^{-6} \AA^{-2}$, $d=500 \AA$.

\subsubsection{Ensemble de couches empilées}

Le formalisme précédent se généralise pour un nombre quelconque de couches sur le substrat.

On considère $\mathrm{p}$ couches déposées sur un substrat $\mathrm{s}$. 


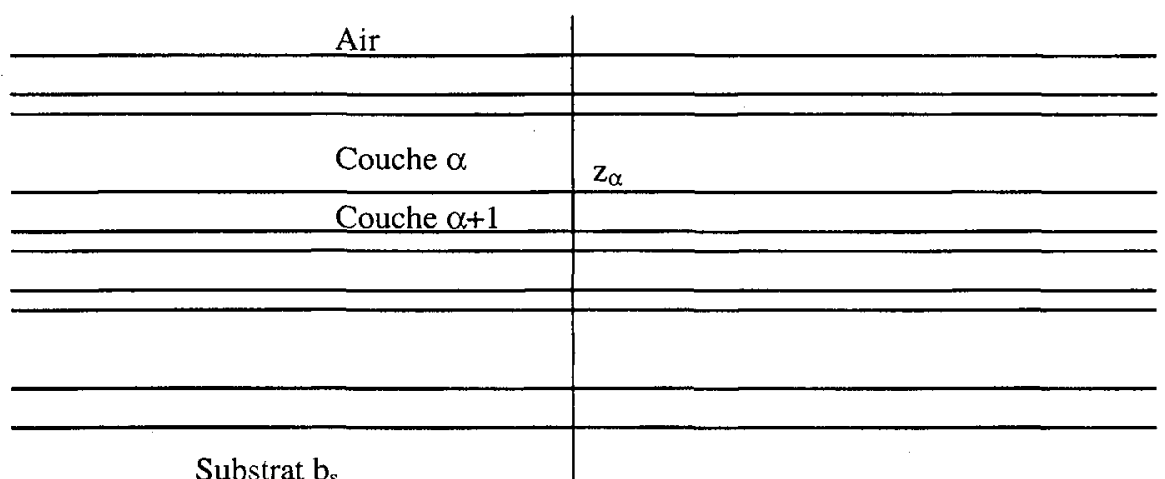

Substrat $b_{s}$

On va définir dans chaque couche, la fonction $\psi$ comme en 29 et les équations de continuité nous permettent d'écrire, pour $\alpha$ variant de 0 à s :

$$
\left(\begin{array}{l}
\mathrm{A}_{\alpha} \\
\mathrm{B}_{\alpha}
\end{array}\right)=\left(\begin{array}{cc}
\left(1+\mathrm{q}_{\alpha+1} / \mathrm{q}_{\alpha}\right) \mathrm{e}^{i \mathrm{q}_{\alpha+1} d_{\alpha}} \mathrm{e}^{-i \mathrm{q}_{\alpha} d_{\alpha} / 2} & \left(1-\mathrm{q}_{\alpha+1} / \mathrm{q}_{\alpha}\right) \mathrm{e}^{-i \mathrm{q}_{\alpha+1} d_{\alpha}} \mathrm{e}^{-i \mathrm{q}_{\alpha} d_{\alpha} / 2} \\
\left(1-\mathrm{q}_{\alpha+1} / \mathrm{q}_{\alpha}\right) \mathrm{e}^{i q_{\alpha+1} d_{\alpha}} \mathrm{e}^{i \mathrm{q}_{\alpha} d_{\alpha} / 2} & \left(1+\mathrm{q}_{\alpha+1} / \mathrm{q}_{\alpha}\right) \mathrm{e}^{i \mathrm{q}_{\alpha+1} d_{\alpha}} \mathrm{e}^{-i \mathrm{q}_{\alpha} d_{\alpha} / 2}
\end{array}\right)\left(\begin{array}{c}
\mathrm{A}_{\alpha+1} \\
\mathrm{~B}_{\alpha+1}
\end{array}\right)
$$

ou

$$
\left(\begin{array}{l}
\mathrm{A}_{\alpha} \\
\mathrm{B}_{\alpha}
\end{array}\right)=\mathbf{M}_{\alpha}\left(\begin{array}{l}
\mathrm{A}_{\alpha+1} \\
\mathrm{~B}_{\alpha+1}
\end{array}\right)
$$

De là on tire par récurrence :

$$
\left(\begin{array}{l}
\mathrm{A}_{0} \\
\mathrm{~B}_{0}
\end{array}\right)=\mathbf{M}_{0} \ldots \mathbf{M}_{\alpha} \ldots \mathbf{M}_{\mathrm{p}}\left(\begin{array}{l}
\mathrm{A}_{s} \\
\mathrm{~B}_{s}
\end{array}\right)=\mathbf{M}\left(\begin{array}{l}
\mathrm{A}_{s} \\
\mathrm{~B}_{s}
\end{array}\right)=\left(\begin{array}{ll}
M_{11} & M_{21} \\
M_{12} & M_{22}
\end{array}\right)\left(\begin{array}{l}
\mathrm{A}_{s} \\
\mathrm{~B}_{s}
\end{array}\right)
$$

La réflectivité est donnée par :

$$
\mathbf{R}=\left|\frac{M_{12}}{M_{11}}\right|^{2}
$$

Pour chaque valeur de $\mathrm{q}_{0}=\mathrm{Q} / 2$, on calcule la fonction $\mathrm{R}$ ci-dessus et on a le profil obtenu expérimentalement.

\section{La réflectivité de neutrons magnétiques}

Dans le cas où l'on tient compte du spin du neutron, la notion d'indice devient hasardeuse et il est nécessaire de remonter à l'équation de Schrödinger que l'on résout dans chaque couche. Je résume ici le calcul permettant la résolution de ce cas. Ce qu'il faut retenir de ce calcul (pour ceux qui veulent le sauter) est que l'utilisation de neutrons polarisés et l'analyse du spin après réflexion permet de remonter non seulement au profil chimique mais aussi au profil vectoriel magnétique de la composante planaire de l'aimantation. C'est actuellement la seule technique qui permet de réaliser cela. 


\subsection{Interaction du neutron avec une couche mince infinie et homogène}

La configuration de la réflexion est identique à celle traitée au dessus, $\mathrm{z}$ étant perpendiculaire au plan de la couche. On va faire l'intégration directe de l'interaction magnétique dans le cas d'une couche mince infinie.

\subsubsection{L'interaction magnétique}

$$
-g_{n} \mu_{n} \sigma . \mathbf{H}=-g_{n} \mu_{n} \sigma .\left[\nabla \times\left\{\frac{\mu_{e} \times \mathbf{r}}{r^{3}}\right\}-\frac{e}{c} \frac{\mathbf{v}_{e} \times \mathbf{r}}{r^{3}}\right]
$$

ou

$$
-g_{n} \mu_{n}\left\{2 \mu_{B} \boldsymbol{\sigma} . \nabla \times\left\{\frac{\mu_{e} \times \mathbf{r}}{r^{3}}\right\}-\frac{e}{2 m_{e} c}\left(\mathbf{p}_{e} \cdot \frac{\boldsymbol{\sigma} \times \mathbf{r}}{r^{3}}+\frac{\boldsymbol{\sigma} \times \mathbf{r}}{r^{3}} \cdot \mathbf{p}_{e}\right\}\right.
$$

avec

$$
\mathbf{p}_{e}=-i \hbar \nabla_{e}
$$

On s'intéresse à la partie de l'interaction avec le spin, l'intégration sur la partie orbitale étant identique. On rappelle que

$$
\begin{aligned}
\nabla \times\left(\frac{\boldsymbol{\mu}_{e} \times \mathbf{r}}{r^{3}}\right)= & -\nabla \times\left(\mu_{e} \times \nabla\left(\frac{1}{r}\right)\right) \\
& =\frac{1}{2 \pi^{2}} \int \frac{1}{q^{2}}\left(\mathbf{q} \times\left(\mu_{e} \times \mathbf{q}\right)\right) \exp (i \mathbf{q} . \mathbf{r}) d \mathbf{q}
\end{aligned}
$$

\subsubsection{Intégration sur une couche homogène}

On fait l'intégration sur la couche de la formule (40) en supposant une densité $\rho$ donnée.

On écrit $\mathbf{r}$ sous la forme, $\mathbf{r}+\mathbf{r}_{0}$. où $\mathbf{r}_{0}$ est la distance de l'origine prise au centre de la couche au neutron et $\mathbf{r}$ est la distance entre l'origine et un volume dr dans la couche.

La partie de l'interaction dépendant du spin s'écrit alors :

$$
2 g_{n} \mu_{n} \mu_{B} \sigma \cdot \frac{1}{2 \pi^{2}} \int_{q^{2}} \frac{1}{V} \rho(\mathbf{r})(\mathbf{q} \times \overline{\mathbf{s}}(\mathbf{r}) \times \mathbf{q}) \exp \left(i \mathbf{q} \cdot \mathbf{r}_{\mathbf{0}}\right) \exp (i \mathbf{q} . \mathbf{r}) d \mathbf{r} d \mathbf{q}
$$

où $\rho(\mathbf{r})$ est la densité à $\mathbf{r}$ and $\overline{\mathbf{s}}(\mathbf{r})=\overline{\mathbf{s}}$ est la valeur moyenne de l'aimantation due au spin par élément de volume dr.

Cela s'écrit aussi :

$$
2 \mu_{N} g_{n} \mu_{B} \sigma \cdot \frac{1}{2 \pi^{2}} \int \frac{1}{q^{2}} \int_{-\infty}^{\infty} \exp \left(i q_{x} r_{x}\right) d r_{x} \int_{-\infty}^{\infty} \exp \left(i q_{y} r_{y}\right) d r_{y} \int_{-d / 2}^{d / 2} \rho d r_{z}(\mathbf{q} \times \overline{\mathbf{s}} \times \mathbf{q}) \exp \left(i \mathbf{q} \cdot \mathbf{r}_{\mathbf{0}}\right) \exp \left(i q_{z} r_{z}\right) d \mathbf{q}
$$


Les deux premières intégrations donnent des fonctions $\delta$.

$$
4 \mu_{N} g_{n} \mu_{B} \rho \boldsymbol{\sigma} \cdot \int \frac{1}{q_{z}^{2}} \int_{-d / 2}^{d / 2} d r_{z}\left(\mathbf{q}_{z} \times \overline{\mathbf{s}} \times \mathbf{q}_{z}\right) \exp \left(i \mathbf{q}_{z} \cdot \mathbf{r}_{\mathbf{0}_{z}}\right) \exp \left(i q_{z} r_{z}\right) d q_{z}
$$

Ce qui donne :

$$
8 \pi \mu_{N} g_{n} \mu_{B} \rho \sigma \cdot \overline{\mathbf{s}}_{/ /}\left[\theta\left(r_{0 z}+d / 2\right)-\theta\left(r_{0 z}-d / 2\right)\right]
$$

Le même calcul peut se faire sur la partie orbitale et donne au total:

$$
\frac{2 \pi \hbar^{2}}{m} p \rho \sigma \cdot \mathbf{M}_{s /}\left[\theta\left(r_{0 z}+d / 2\right)-\theta\left(r_{0 z}-d / 2\right)\right]
$$

avec $\mathrm{p}=0.269610^{-12} \mathrm{~cm}$

$\theta$ est la fonction marche définie par :

$$
\begin{aligned}
& \theta(x)=1 \text { quand } x>0 \\
& \theta(x)=1 / 2 \text { quand } x=0 \\
& \theta(x)=0 \text { quand } x<0
\end{aligned}
$$

$\mathbf{M}_{/ /}$est donnée en unités de $\mu_{\mathrm{B}}$ et représente la composante dans le plan et pas nécessairement la composante perpendiculaire au vecteur de diffusion

\subsubsection{Conclusions}

De la forme de (44) on peut en tirer que

- On ne peut mesurer que la projection de l'aimantation dans le plan.

- L'interaction du neutron et du plan magnétique est nulle dès que le neutron est hors de ce plan.

Ces deux propriétés sont essentielles. La première est la principale et peut-être la seule limitation des neutrons pour l'étude des couches minces. La seconde justifie de pouvoir résoudre l'équation de Schrödinger dans une couche en oubliant qu'il y a d'autres couches magnétiques autour. Le formalisme utilisé pour les couches non magnétiques pourra être appliqué au cas des couches magnétiques.

\subsection{Quelques remarques sur la précession du neutron, réseau artificiel}

On considère une couche mince attaquée en angle rasant par un neutron précessant autour du champ magnétique extérieur.

Dans ce cas lorsque l'onde rencontre le début du cristal, son spin pointe dans une certaine direction qui sera différente de la partie arrivant à la fin du cristal : l'onde est réfléchie différemment en 
fonction de la position d'arrivée. Tout se passe comme si on diffusait sur un réseau artificiel harmonique de période $d=v .2 \pi / g_{n} \mu_{n} \mathbf{H}_{0}=34 \mathrm{~cm} /$ Gauss

Cela conduit à une séparation de l'onde réfléchie et transmise en deux faisceaux distincts.

Dans toute la suite, on va considérer un champ magnétique le long d'une direction quelconque, $z$ désigne encore la perpendiculaire au plan de l'échantillon.

\subsection{Equation de Schrödinger}

Le potentiel d'interaction dans une couche $\alpha$ donnée est :

$$
V_{\alpha}=\frac{2 \pi \hbar^{2}}{m} \rho_{\alpha} b_{\alpha}-\frac{2 \pi \hbar^{2}}{m} p \sigma \cdot \rho \mathbf{M}_{\alpha / \prime}-g_{n} \mu_{n} \sigma . \mathbf{H}_{0}
$$

Ce potentiel est constant donc le problème est soluble. On a un ensemble de deux équations :

$$
\begin{aligned}
& \left.\left(-\frac{\hbar^{2}}{2 m} \nabla^{2}+\frac{2 \pi \hbar^{2}}{m} b_{\alpha} \rho_{\alpha}-g_{n} \mu_{n} \mathbf{H}_{0 z}\right) \psi_{+}(\mathbf{r})+\left(-g_{n} \mu_{n} \mathbf{H}_{0 x}+\frac{2 \pi \hbar^{2}}{m} p \rho_{\alpha} M_{\alpha x}+i g_{n} \mu_{n} \mathbf{H}_{0 y}-i \frac{2 \pi \hbar^{2}}{m} p \rho_{\alpha} \mathbf{M}_{\alpha y}\right)\right) \psi_{-}(\mathbf{r})=E \psi_{+}(\mathbf{r}) \\
& \left.\left(-\frac{\hbar^{2}}{2 m} \nabla^{2}+\frac{2 \pi \hbar^{2}}{m} b_{\alpha} \rho_{\alpha}+g_{n} \mu_{n} \mathbf{H}_{0 z}\right) \psi_{-}(\mathbf{r})+\left(-g_{n} \mu_{n} \mathbf{H}_{0 x}+\frac{2 \pi \hbar^{2}}{m} p \rho_{\alpha} M_{\alpha x}-i g_{n} \mu_{n} \mathbf{H}_{0 y}+i \frac{2 \pi \hbar^{2}}{m} p \rho_{\alpha} \mathbf{M}_{\alpha y}\right)\right) \psi_{+}(\mathbf{r})=E \psi_{-}(\mathbf{r})
\end{aligned}
$$

(équation 46)

\subsection{Solution générale}

On cherche des solutions de la forme : $\psi_{+}(\mathbf{r})=a_{+} \exp (i \mathbf{k} . \mathbf{r})$ et $\psi_{-}(\mathbf{r})=a_{-} \exp (i \mathbf{k} . \mathbf{r})$

Les valeurs possibles pour $q$ sont données par la nullité du déterminant du système ci-dessus :

où

$$
k_{\alpha}^{ \pm 2}=\frac{2 m}{\hbar^{2}} E-4 \pi \rho_{\alpha} b_{\alpha} \pm \frac{2 m g_{n} \mu_{n}}{\hbar^{2}}\left|H_{\alpha}\right|
$$

$$
\left\{\begin{array}{l}
H_{\alpha x}=H_{0 x}-\frac{2 \pi \hbar^{2}}{g_{n} \mu_{n} m} p \rho_{\alpha} M_{\alpha x}=H_{0 x}-4 \pi \mu_{B} \rho_{\alpha} M_{\alpha x}=H_{\alpha} \sin \left(\theta_{\alpha}\right) \cos \left(\varphi_{\alpha}\right) \\
H_{y}=H_{0 y}-\frac{2 \pi \hbar^{2}}{g_{n} \mu_{n} m} p \rho_{\alpha} M_{\alpha y}=H_{0 y}-4 \pi \mu_{B} \rho_{\alpha} M_{\alpha y}=H_{\alpha} \sin \left(\theta_{\alpha}\right) \sin \left(\varphi_{\alpha}\right) \\
H_{z}=H_{0 z}=H_{\alpha} \cos \left(\theta_{\alpha}\right)
\end{array}\right.
$$

est le champ effectif vu par le neutron.

La symétrie du problème fait que les gens prennent une onde de la forme :

$$
\exp \left(i \mathbf{k}_{\alpha \prime \prime}, \mathbf{r}_{\prime \prime}\right) \cdot\left(a \exp \left(i q_{\alpha z}^{+} \cdot z\right)+b \exp \left(-i q_{\alpha z}^{+} \cdot z\right)+c \exp \left(i q_{\alpha z}^{-} \cdot z\right)+d \exp \left(-i q_{\alpha z}^{-} \cdot z\right)\right)(49)
$$

Le seul problème est que cela n'est valide que si $q_{0 / 1}^{+}=q_{0 / 1}^{-}=q_{\alpha / 1}^{+}=q_{\alpha / /}^{-}$ce qui n'est en général pas le cas. 
Pratiquement, la solution est de forme plus compliquée :

$$
\begin{aligned}
& \exp \left(i \mathbf{k}_{\alpha / f}^{++}, \mathbf{r}_{f \prime}\right) \cdot\left(a \exp \left(i q_{o c}^{++} \cdot z\right)+b \exp \left(-i q_{\alpha z}^{++} \cdot z\right)\right)+\exp \left(i \mathbf{k}_{\alpha f}^{-}, \mathbf{r}_{t \prime}\right) \cdot\left(c \exp \left(i q_{o z}^{--} \cdot z\right)+d \exp \left(-i q_{\alpha z}^{--} \cdot z\right)\right)+
\end{aligned}
$$

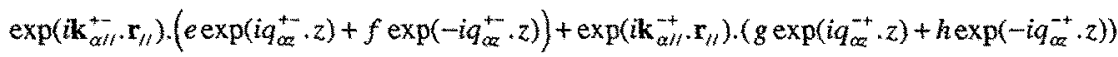

$$
\begin{aligned}
& \operatorname{avec} \begin{cases}k_{\alpha}^{++2}=k_{\alpha}^{+2}, & k_{\alpha / \prime}^{++2}=k_{0 / 1}^{+2} \\
k_{\alpha}^{+-2}=k_{\alpha}^{+2}, & k_{\alpha \prime \prime}^{+-2}=k_{0 / t}^{-2} \\
k_{\alpha}^{+2}=k_{\alpha}^{-2}, & k_{\alpha / !}^{+2}=k_{0 / \prime}^{+2} \\
k_{\alpha}^{--2}=k_{\alpha}^{-2}, & k_{\alpha \prime \prime}^{-2}=k_{0 / \prime}^{-2}\end{cases}
\end{aligned}
$$

La solution générale de l'équation (46) est donc la solution (50) tournée le long des axes de quantification :

$$
\begin{aligned}
& \psi_{\alpha}^{+}(\mathbf{r})=\exp \left(i \mathbf{k}_{\alpha / /}^{++} \mathbf{r}_{/ /}\right)\left(a_{\alpha}^{++} \exp \left(i q_{\alpha z}^{++} z\right)+b_{\alpha}^{++} \exp \left(-i q_{\alpha z}^{++} z\right)\right) \cdot \cos \left(\theta_{\alpha} / 2\right) \\
& \exp \left(i \mathbf{k}_{\alpha / /}^{+-} \mathbf{r}_{t /}\right)\left(a_{\alpha}^{+-} \exp \left(i q_{\alpha z}^{+-} z\right)+b_{\alpha}^{+-} \exp \left(-i q_{\alpha z}^{+-} z\right)\right) \cdot \cos \left(\theta_{\alpha} / 2\right) \\
& -\exp \left(i \mathbf{k}_{\alpha / /}^{-+} \mathbf{r}_{/ \prime}\right)\left(a_{\alpha}^{-+} \exp \left(i q_{\alpha z}^{-+} z\right)+b_{\alpha}^{-+} \exp \left(-i q_{\alpha z}^{-+} z\right)\right) \cdot \mathrm{e}^{-i \varphi_{u}} \sin \left(\theta_{\alpha} / 2\right) \\
& -\exp \left(i \mathbf{k}_{\alpha / l}^{-} \mathbf{r}_{/ /}\right)\left(a_{\alpha}^{--} \exp \left(i q_{\alpha z}^{--} z\right)+b_{\alpha}^{--} \exp \left(-i q_{\alpha z}^{--} z\right)\right) \cdot \mathrm{e}^{-i \varphi_{u}} \sin \left(\theta_{\alpha} / 2\right) \\
& \psi_{\alpha}^{-}(\mathbf{r})=\exp \left(i \mathbf{k}_{\alpha / /}^{++} \mathbf{r}_{l \prime}\right)\left(a_{\alpha}^{++} \exp \left(i q_{\alpha z}^{++} z\right)+b_{\alpha}^{++} \exp \left(-i q_{\alpha z}^{++} z\right)\right) \cdot \mathrm{e}^{i \varphi_{\alpha z}} \sin \left(\theta_{\alpha} / 2\right) \\
& \exp \left(i \mathbf{k}_{\alpha / /}^{+-} \mathbf{r}_{\| \prime}\right)\left(a_{\alpha}^{+-} \exp \left(i q_{\alpha z}^{+-} z\right)+b_{\alpha}^{+-} \exp \left(-i q_{\alpha z}^{+-} z\right)\right) \cdot \mathrm{e}^{i \varphi_{a t}} \sin \left(\theta_{\alpha} / 2\right) \\
& +\exp \left(i \mathbf{k}_{\alpha / /}^{-+} \mathbf{r}_{j /}\right)\left(a_{\alpha}^{-+} \exp \left(i q_{\alpha z}^{-+} z\right)+b_{\alpha}^{-+} \exp \left(-i q_{\alpha z}^{-+} z\right)\right) \cdot \cos \left(\theta_{\alpha} / 2\right) \\
& +\exp \left(i \mathbf{k}_{\alpha / f}^{-} \mathbf{r}_{y \prime}\right)\left(a_{\alpha}^{--} \exp \left(i q_{\alpha z}^{--} z\right)+b_{\alpha}^{--} \exp \left(-i q_{\alpha z}^{--} z\right)\right) \cdot \cos \left(\theta_{\alpha} / 2\right)
\end{aligned}
$$

\subsection{Conditions aux limites et formalisme matriciel}

Les huit constantes $a_{\alpha}^{\ddagger \pm}$ et $b_{\alpha}^{ \pm \pm}$sont déterminées en utilisant la continuité de $\psi$ and $\nabla \psi$ à l'interface de deux couches. En fait, cela donne exactement 8 équations

Le problème se simplifie notablement en remarquant que la continuité doit être vraie quelque soit $r_{/ \prime}$ et donc le système se sépare en deux groupes : le groupe avec les exposants ${ }^{++}$et $^{++}$qui correspondent à $k_{0 / t}^{+}$et le groupe avec les exposants ${ }^{+*}$ et “ qui correspondent à $k_{0 / t}^{-}$

On retrouve la loi bien connue : le résultat obtenu pour la réflexion ou la transmission d'un neutron qui n'est pas dans un état propre est la somme pondérée des réflexion et transmission des états propres.

Pour traiter le problème, on utilise un traitement matriciel identique à celui de neutrons non polarisés. Mais ici les équations de continuitể ne conduisent pas à 2 équations mais 8 équations. Ainsi, les relations de continuités s'écrivent : 


$$
\underline{D}_{\alpha}\left(\overrightarrow{\mathbf{r}}_{\alpha}\right)\left(\begin{array}{c}
a_{\alpha}^{++} \\
b_{\alpha}^{++} \\
a_{\alpha}^{-+} \\
b_{\alpha}^{-+} \\
a_{\alpha}^{--} \\
b_{\alpha}^{--} \\
a_{\alpha}^{+-} \\
b_{\alpha}^{+-}
\end{array}\right)=D_{\alpha+1}\left(\overrightarrow{\mathbf{r}}_{\alpha}\right)\left(\begin{array}{c}
a_{\alpha+1}^{++} \\
b_{\alpha+1}^{++} \\
a_{\alpha+1}^{-+} \\
b_{\alpha+1}^{-+} \\
a_{\alpha+1}^{--1} \\
b_{\alpha+1}^{-} \\
a_{\alpha+1}^{+-} \\
b_{\alpha+1}^{+-}
\end{array}\right)
$$

La matrice $\underline{D}_{\alpha}\left(\vec{r}_{\alpha}\right)$, de dimension $8 \times 8$, s'écrit en fonction de blocs matriciels de dimensions $4 \times 4$ :

$$
\underline{\mathrm{D}}_{\alpha}\left(\overrightarrow{\mathbf{r}}_{\alpha}\right)=\left(\begin{array}{cc}
\underline{\mathrm{DA}}_{\alpha} & \underline{0} \\
\underline{0} & \underline{\mathrm{DB}}_{\alpha}
\end{array}\right)
$$

Par souci de clarté, dans les équations (53), on prendra les notations suivantes :

$\left(k_{i f}^{l i}=k_{\alpha \prime \prime}^{i i}\right.$ avec $\mathrm{i}=+$ ou -$)$ et $\left(\mathrm{r}=\mathrm{r}_{\alpha / l}\right)$ et $\left(\mathrm{q}_{\mathrm{z}}^{\mathrm{ii}}=\mathrm{q}_{\alpha \mathrm{z}}^{\mathrm{ii}}\right.$ avec $\mathrm{i}=+$ ou -$)$ et $\left(\mathrm{z}=\mathrm{z}_{\alpha}\right)$ et $\left(\theta^{\prime}=\frac{\theta_{\alpha}}{2}\right)$ et $\left(\varphi=\varphi_{\alpha}\right)$

\section{$\underline{\mathrm{DA}}_{\alpha}=$}

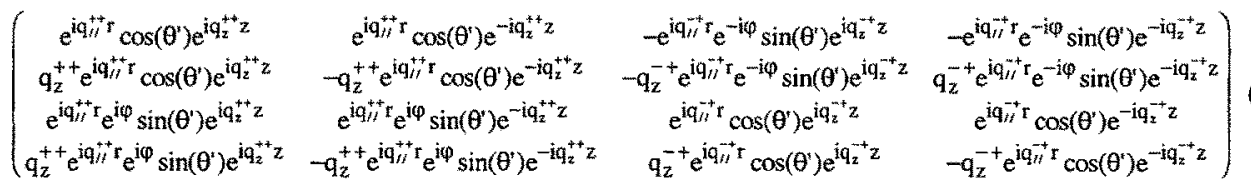

$\underline{\mathrm{DB}}_{\alpha}=$

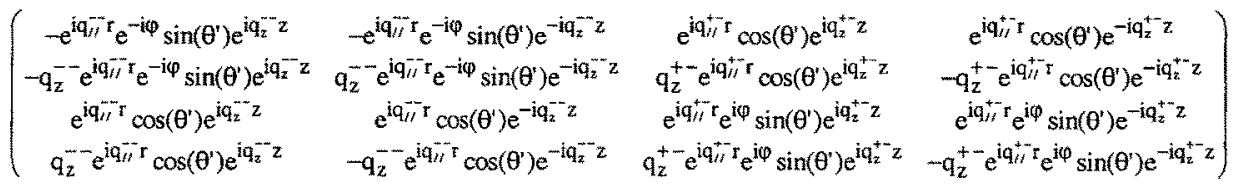

La matrice de transfert $\underline{M}$, de dimension $8 \times 8$, est définie par :

$$
\underline{\mathrm{M}}=\prod_{\alpha=0}^{\alpha=\mathrm{N}} \underline{\mathrm{D}}_{\alpha}^{-1}\left(\overrightarrow{\mathbf{r}}_{\alpha}\right) \underline{\mathrm{D}}_{\alpha+1}\left(\overrightarrow{\mathbf{r}}_{\alpha}\right)=\underline{\mathrm{M}}=\left(\begin{array}{cc}
\frac{\mathrm{MA}}{\underline{0}} & \underline{\mathrm{MB}}
\end{array}\right)
$$

où les blocs matriciels sont :

$$
\underline{\mathrm{MA}}=\underline{\mathrm{DA}}_{\alpha}^{-1} \underline{\mathrm{DA}}_{\alpha+1} \text { et } \underline{\mathrm{MB}}=\underline{\mathrm{DB}}_{\alpha}^{-1} \underline{\mathrm{DB}}_{\alpha+1}
$$


Des équations (53) et (57), on obtient finalement la relation : l'indice 0 correspond à un neutron situé dans l'air, l'indice $s$ correspond ici à un neutron situé dans le substrat.

$$
\left(\begin{array}{l}
a_{0}^{++} \\
b_{0}^{++} \\
a_{0}^{-+} \\
b_{0}^{-+} \\
a_{0}^{--} \\
b_{0}^{--} \\
a_{0}^{+-} \\
b_{0}^{+-}
\end{array}\right)=\underline{M}\left(\begin{array}{l}
a_{s}^{++} \\
b_{s}^{++} \\
a_{s}^{-+} \\
b_{s}^{-+} \\
a_{s}^{-+} \\
b_{s}^{--} \\
a_{s}^{+-} \\
b_{s}^{+-}
\end{array}\right)
$$

Envisageons maintenant l'exemple simple d'une expérience de rếflectivité de neutrons où le faisceau incident ne contient que des neutrons polarisés up ou des neutrons polarisés down:

l'équation (59) devient soit, pour des neutrons up:

$$
\left(\begin{array}{l}
1 \\
r_{0}^{++} \\
0 \\
r_{0}^{-+} \\
0 \\
0 \\
0 \\
0
\end{array}\right)=\underline{M}\left(\begin{array}{l}
t_{s}^{++} \\
0 \\
t_{s}^{-+} \\
0 \\
0 \\
0 \\
0 \\
0
\end{array}\right)
$$

soit, pour des neutrons down :

$$
\left(\begin{array}{l}
0 \\
0 \\
0 \\
0 \\
1 \\
r_{0}^{-} \\
0 \\
r_{0}^{+-}
\end{array}\right)=\underline{M}\left(\begin{array}{l}
0 \\
0 \\
0 \\
0 \\
t_{s}^{--} \\
0 \\
t_{s}^{+\cdots} \\
0
\end{array}\right)
$$

On appelle $\mathrm{r}_{0}^{++}, \mathrm{r}_{0}^{-+}$les coefficients de réflectivité dans l'air pour un neutron incident up, réfléchi up (resp. down), les coefficients de transmission dans le substrat sont notés $\mathrm{t}_{\mathrm{N}}^{++}, \mathrm{t}_{\mathrm{N}}^{+}$.

De l'équation (22a), on en déduit les expressions de chacun des coefficients : 


$$
\left\{\begin{array} { l } 
{ r _ { 0 } ^ { + + } = \frac { M _ { 2 1 } \mathrm { MA } _ { 3 3 } - \mathrm { MA } _ { 2 3 } \mathrm { MA } _ { 3 1 } } { \mathrm { MA } _ { 1 1 } \mathrm { MA } _ { 3 3 } - \mathrm { MA } _ { 1 3 } \mathrm { MA } _ { 3 1 } } } \\
{ \mathrm { r } _ { 0 } ^ { - + } = \frac { \mathrm { MA } _ { 4 1 } \mathrm { MA } _ { 3 3 } - \mathrm { MA } _ { 4 3 } \mathrm { MA } _ { 3 1 } } { \mathrm { MA } _ { 1 1 } \mathrm { MA } _ { 3 3 } - \mathrm { MA } _ { 1 3 } \mathrm { MA } _ { 3 1 } } }
\end{array} \quad \left\{\begin{array}{l}
\mathrm{t}_{\mathrm{N}}^{++}=\frac{\mathrm{MA}_{33}}{\mathrm{MA}_{11} \mathrm{MA}_{33}-\mathrm{MA}_{13} \mathrm{MA}_{31}} \\
\mathrm{t}_{\mathrm{N}}^{++}=\frac{-\mathrm{MA}_{31}}{\mathrm{MA}_{11} \mathrm{MA}_{33}-\mathrm{MA}_{13} \mathrm{MA}_{31}}
\end{array}\right.\right.
$$

On trouve des relations comparables pour les quatre autres coefficients de l'équation (71).

L'intensité des neutrons détectés, après réflexion sur la multicouche, dans l'angle solide $\Omega$, est proportionnelle au carré du coefficient de réflectivité de la fonction d'onde du neutron réfléchi; de façon générale, on aura pour les deux états de polarisation du neutron :

$$
\mathrm{I}^{++} \propto\left|\mathrm{r}^{++}\right|^{2} \text { et } \mathrm{I}^{--} \propto\left|\mathrm{r}^{--}\right|^{2} \text { et } \mathrm{I}^{+-} \propto\left|\mathrm{r}^{+-}\right|^{2} \text { et } \mathrm{I}^{++} \propto\left|\mathrm{r}^{-+}\right|^{2}
$$

Remarque : dans le cas de champ magnétique extérieur faible, on $\mathrm{a}^{++}=\mathrm{r}^{-+}$.

\section{Les couches non parfaites, les problèmes pratiques et les limitations expérimentales}

\subsection{Rugositês d'interfaces}

La plupart des systèmes étudiés vont présenter des interfaces imparfaites qui ont pour origine le mode de dépôt de la couche. On parlera de rugosité d'interface, d'interdiffusion ou de planéité de la couche suivant les échelles latérales que nous considérerons.

Dans le langage des couches minces solides, on distinguera trois origines d'imperfections :

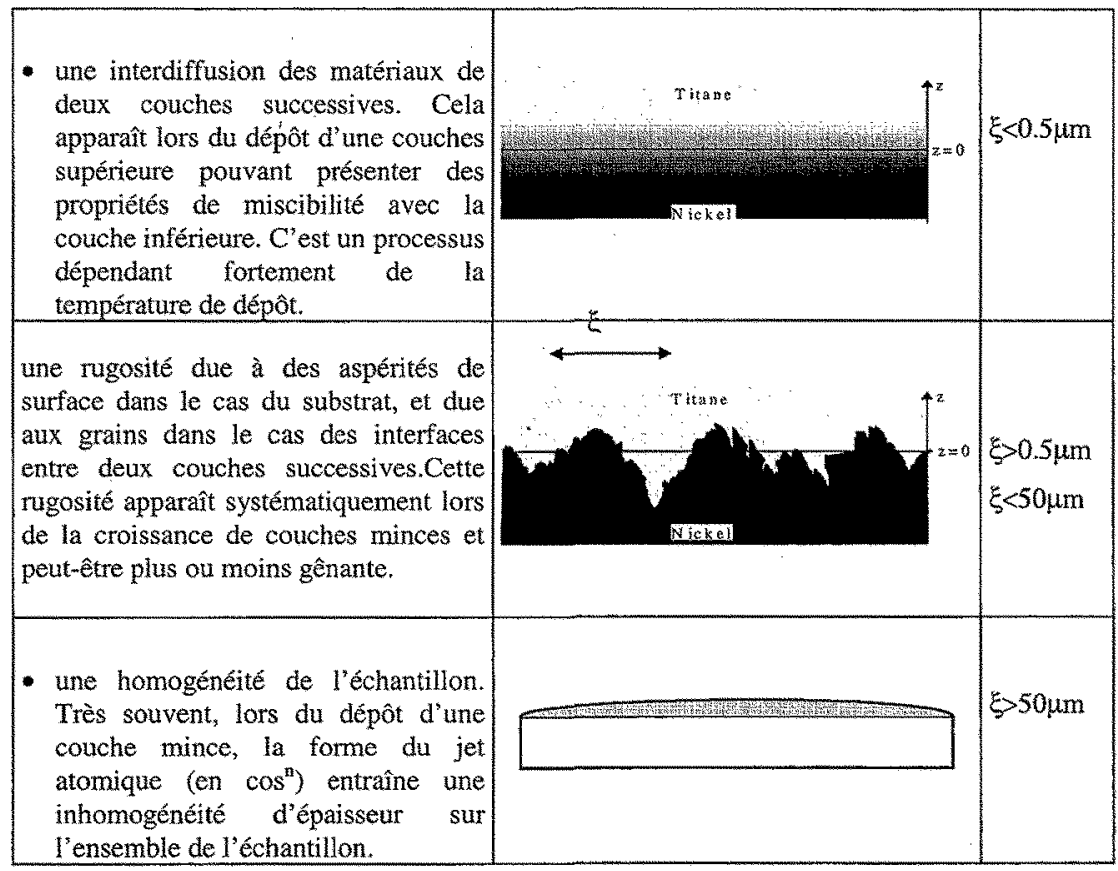


Pour les neutrons, on fera le même type de distinction mais la limite ne sera pas donnée par la nature (rugosité ou interdiffusion) mais par la période latérale considérée $\xi$, les ordre de grandeur sont donnés dans le tableau ci-dessus.

Ces trois types d'imperfections seront traitées différemment pour ajuster une courbe de réflectivité. Ils ont d'ailleurs des influences très différentes sur les courbes.

Il y a une limitation dont il faut se souvenir. Si les fluctuations latérales ne sont pas faibles devant l'épaisseur des couches envisagées, toutes les méthodes dont je vais parler ensuite ne marcheront pas bien.

\subsubsection{Inhomogénéité de l'échantillon}

Il n'existe la pas de méthode miracle pour résoudre le problème. La courbe expérimentale est simplement la somme des différentes courbes théoriques avec des épaisseurs différentes. L'effet de ce type de défaut est un brouillage des cohérences à grand $\mathrm{Q}$.

La figure 5 illustre bien cet effet.

Dans un programme de simulation (bien fait), on pourra introduire pour chaque couche, une fluctuation d'épaisseur et en plus une fluctuation globale de l'épaisseur de la multicouche.

La fluctuation globale de l'épaisseur se traite comme une divergence angulaire (voir plus loin).

\subsubsection{Rugosité et interdiffusion}

$\mathrm{Au}$ premier abord, la réflectivité spéculaire de neutrons ne peut distinguer les deux types d'interfaces, rugosités ou diffusion. En effet, la mesure de la valeur de la densité de longueur de diffusion cohérente $\rho b$ se fait sur une grande distance par rapport à la taille des aspérités : en une cote $\mathrm{z}$ donnée, on mesure une valeur de $\rho b$ moyennée sur une grande distance.

\subsubsection{Première solution: introduction d'un facteur de Debve-Waller}

Dans la mesure où on suppose, une distribution plate des valeurs de $\xi$, les deux types d'interfaces peuvent être regroupées dans un modèle unique où la fonction escalier est remplacée par la fonction erreur suivante :

$$
\operatorname{erf}\left(\frac{z-z_{\alpha}}{\sigma_{\alpha}}\right)=\frac{2}{\sqrt{\pi}} \int_{0}^{\left(z-z_{u}\right) / \sigma_{\alpha}} \mathrm{e}^{-t^{z}} d t
$$

La courbe représentative de cette fonction erreur présente un point dinflexion en $z_{\alpha}$. La valeur $\sigma_{\alpha}$ est donnée par l'inverse de la pente de la tangente à la courbe en $\mathrm{z}_{\alpha}$. L'épaisseur de l'interface est donnée par $2 \sigma_{\alpha}$

Le résultat de (70) est de multiplier la fonction $\mathrm{R}$ calculée pour une interface définie par les milieux $\alpha$ et $\alpha+1$ par un facteur de type Debye-Waller de la forme :

$$
D W=\exp \left(-4 q_{\alpha} q_{\alpha+l} \sigma_{\alpha}^{2}\right)
$$

avec $\mathrm{q}_{\alpha}$ et $\mathrm{q}_{\alpha+1}$ définis par l'équation (31). 
Dans le cas d'un empilement multicouches dont chaque interface présente une rugosité propre, le facteur DW intervient dans chaque matrice. Malheureusement, cette technique est difficilement applicable dans le cas du magnétisme, le formalisme ne nous permettant pas de tirer aisément la réflectivité $\mathrm{R}$ à chaque interface.

On peut cependant contourner partiellement le problème d'une part en introduisant un facteur DW global et ensuite en introduisant un tel facteur sur les éléments diagonaux à chaque interface. Pratiquement cela marche pas mal.

L'effet le plus direct d'un tel facteur est bien sur de diminuer la réflectivité pour de grandes valeurs de Q.

\subsubsection{2 seconde solution, la discrétisation}

Cette technique permet de bien traiter l'interdiffusion.

On remplace l'interface par un nombre fini de couches discrètes présentant un gradient de concentration. On peut utiliser un profil en fonction d'erreur avec des résultats s'approchant de a), soit des systèmes linéaires. Pratiquement, pour des systèmes solides usuels, une seule couche avec un $\rho b$ moyen marche pas mal.

La figure 5 montre les effets de la rugosité sur la courbe de réflectivité d'une couche de nickel déposée sur un substrat de silicium. Les rugosités $\sigma_{\mathrm{s}}$ et $\sigma_{\mathrm{air}}$ varient d'une courbe à l'autre comme indiqué dans la légende. On remarque sur cette courbe que les rugosités provoquent une diminution du signal à grands q, ainsi qu'une diminution de l'amplitude des franges. Ici la rugosité a été traitée au moyen d'une discrétisation des couches.

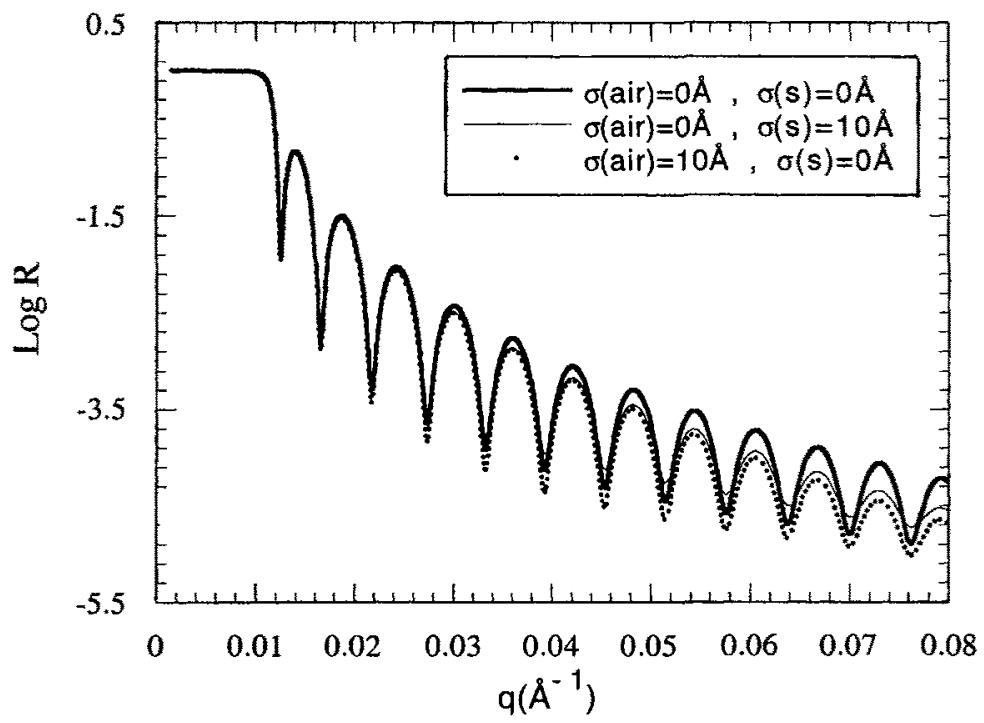

Figure 5: Simulations numériques de l'effet des rugosités $\sigma_{s}$ et $\sigma_{a i r}$ sur la courbe de réflectivité d'une couche de nickel de $500 \AA$ sur un substrat de silicium. Trait épais : Référence, $\sigma_{s}=\sigma_{a i r}=0 \AA$; trait continu fin : effet de $\sigma_{s}$; points : effet de $\sigma_{\text {air. }}$ 


\subsubsection{La rugosité intermédiaire}

Dans le cas de la présence d'une rugosité intermédiaire, les méthodes ci-dessus ne sont pas pleinement satisfaisantes. En effet, l'effet de ce type de rugosité ne consiste pas seulement à diminuer l'intensité de la réflexion spéculaire mais elle crée un fond de diffusion hors spéculaire qui peut brouiller les résultats. Dans le cas d'échantillons de ce type, il faut tracer si possible une courbe de l'intensité hors spéculaire et corriger l'intensité spéculaire en fonction de des résultats. Le traitement de la réflectivité hors spéculaire dépasse largement le contenu de ce cours mais pourra être foumie à ceux qui le désirent. Des mesures AFM sont pour la surface, toujours intéressantes à mesurer car elles renseignent sur la croissance des couches.

\subsubsection{4 la rugosité magnétique}

Là le problème est bien plus ardu.

L'exemple typique de rugosité magnétique est un échantillon désaimanté. Dans ce cas chaque domaine a une longueur de diffusion effective très différente du domaine voisin. Cela apparaît pour le neutron comme une rugosité géante de la couche. On n'a donc pas de moyens théoriques fiables de rendre compte des résultats. Par réflectivité magnétique hors spéculaire, on peut avoir une mesure, dans les bons cas, de la taille moyenne des domaines.

\subsection{Résolution angulaire}

Les expressions de la réflectivité déterminées dans les paragraphes précédents ont été calculées pour des faisceaux incidents sans divergence.

Le faisceau incident présente une certaine divergence $\delta \theta$ qui induit un élargissement du faisceau réfléchi. Le calcul se fait par la convolution de la fonction calculée $R(Q)$ avec une fonction donnée par l'appareil $\mathrm{f}\left(\theta, \delta \theta_{0}\right)$ centrée en 0 . Une fonction carrée marche assez bien, d'autres utilisent une fonction triangle moins bien justifiée.

$$
R_{\delta \theta ; \theta}(Q)=\int R\left(Q \frac{\sin \theta_{0}}{\sin \theta}\right) f\left(\theta_{0}-\theta, \delta \theta\right) d \theta
$$

Remarquons que bien que la valeur de $\delta \theta$ soit fixée par la configuration du spectromètre pour une mesure donnée, sa valeur peut nécessiter un ajustement au cours du traitement des résultats. En effet, les échantillons étudiés, bien que les substrats soient choisis pour être les plus plans possible (pastille de silicium épais, verre flotté), présentent une certaine courbure. Celle-ci a un effet similaire au $\delta \theta$ (élargissement du faisceau), elle est alors intégrée dans la valeur de $\delta \theta$.

Une résolution angulaire $\delta \theta$ non nulle provoque une incertitude sur la valeur de q. Cette erreur sur $\mathrm{q}$ induit :

- Une diminution de l'amplitude du signal (par l'addition des deux courbes décalées calculées à $\mathrm{q} \pm \delta \mathrm{q})$; son effet est un "arrondissement" de la courbe de réflectivité aux points anguleux.

La Figure.6, où est représentée la courbe de réflectivité calculée pour un système à une interface avec variation de la résolution angulaire, illustre ces deux phénomènes :

- A la coupure, on remarque l'arrondi de la courbe.

- A grands q on remarque la baisse de réflectivité. 


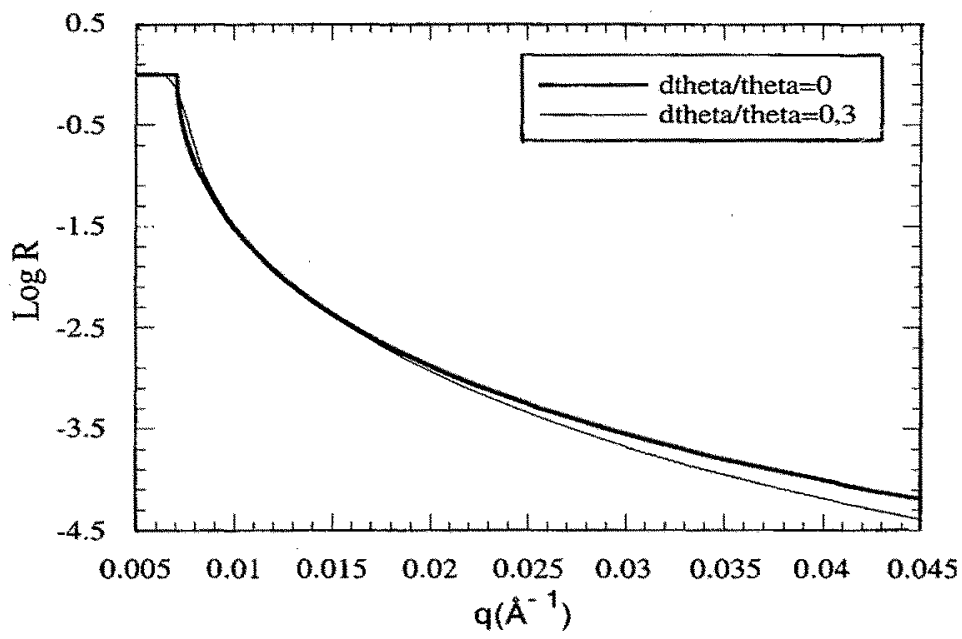

Figure.6 : Simulation de l'effet de la résolution angulaire sur la courbe de réflectivité d'un substrat de verre $\left(\rho b=4 \times 10^{-6} \AA^{-2}\right)$ : trait épais : $\delta \theta / \theta=0 ;$ trait fin : $\delta \theta / \theta=0,3$.

\subsection{Méthode d'analyse des données expérimentales}

\subsubsection{Traitement des données par simulation numérique}

Le traitement des courbes expérimentales de réflectivité de neutrons se fait par ajustement numérique de la courbe calculée pour le système multicouche considéré, à la courbe expérimentale. Les paramètres d'ajustement sont les épaisseurs des couches, les densités données en $a t / \mathrm{cm}^{3}$, les longueurs de diffusion cohérentes b de chaque matériau, les rugosités d'interfaces et les moments pour les couches magnétiques, en intensité et en angle.

\subsubsection{Problème de l'inversion des données et d'unicité des solutions}

Contrairement à la transformée de Fourrier, les courbes de réflectivité ne sont pas inversibles. On peut même montrer théoriquement, dans le cas de systèmes non magnétiques, que le fait de ne pas connaître la phase de la réflectivité (on ne mesure que l'intensité) permet de construire à partir d'une solution donnée de construire toute une famille de profils donnant le même résultat. Pour le magnétisme, dans la mesure où le flux de neutrons serait suffisant, les ajustements sur diverses configurations de spin permet d'éviter ce problème.

Il reste tout de même et surtout les incertitudes dues au manque de signal chronique des expériences de réflectivité aux neutrons. 


\section{Les Réflectomètres, la réflectivité $X$}

\subsection{Introduction}

Dans les pages qui suivent sont présentés quelques réflectomètres à neutrons. Une description assez détaillé du réflectomètre à temps de vol EROS du LLB est donnée. Une description plus sommaire du réflectomètre à temps de vol CRISP de ISIS, ainsi que du réflectomètre à longueur d'onde fixe PADA du LLB est également présentée.

\subsection{Principes de la mesure d'une courbe de réflectivité}

Le principe du calcul du coefficient de la réflectivité est simple : il s'agit de diviser lintensité du faisceau réfléchi par un échantillon, par l'intensité du faisceau direct éclairant l'échantillon. Ces intensités sont mesurées pour chaque valeur de $Q=\frac{4 \pi \sin \theta}{\lambda}$. Si le faisceau de neutrons qui éclaire l'échantillon est polychromatique, la valeur de chaque longueur d'onde est déterminée au moyen de la technique de temps de vol. ${ }^{4,5}$ Les réflectomètres EROS du Laboratoire Léon Brillouin (LLB) au CE Saclay et CRISP du Rutherford Appleton Laboratory à Didcot, Grande-Bretagne utilisent cette méthode. La seconde méthode consiste à faire varier l'angle $\theta$ en travaillant avec un faisceau monochromatique. C'est le cas du réflectomètre en neutrons polarisés PADA du LLB.

\subsection{La technique de temps de vol}

La technique du temps de vol permet de séparer les neutrons d'un faisceau blanc en fonction de leur vitesse. Elle est basée sur la relation de de Broglie selon laquelle le temps $t$ qu'un neutron met pour parcourir la distance L est proportionnel à sa longueur d'onde associée $\lambda$ :

$$
\lambda=\frac{\mathrm{h}}{\mathrm{mL}} \mathrm{t}
$$

où h est la constante de Plank et m est la masse du neutron. Cette relation est équivalente à :

$$
\lambda(\dot{A})=\frac{t(\mu s)}{252,7 L(m)}
$$

Un dispositif de hacheur permet de former des bouffées de neutrons contenant toutes les longueurs d'onde disponibles. Chaque bouffée est formée au temps $t=0$, et la mesure du temps nécessaire aux neutrons pour aller du hacheur au détecteur (distance L) permet de déterminer leur longueur d'onde. La vitesse de rotation du hacheur est telle que les neutrons les plus lents d'une bouffée arrivent avant les neutrons les plus rapides de la bouffée suivante. Cette méthode permet de déterminer la distribution en longueur d'onde des faisceaux incidents et téfléchis. 


\subsection{Les réflectomètres}

\section{EROS}

Les neutrons produits par le réacteur Orphée sont thermalisés par une source froide d'hydrogène liquide à une température de l'ordre de $20 \mathrm{~K}$, puis amenés jusqu'au réflectomètre par un guide de neutrons. En fin du guide, on dispose d'un faisceau polychromatique dont les longueurs d'onde sont comprises entre 3 et $25 \AA$. La Figure 7 schématise le réflectomètre EROS. Le hacheur est constitué d'un disque sur la périphérie duquel se trouve une fente. Ce disque, fait d'un matériau absorbeur de neutrons, laisse passer ces demiers par la fente et crée ainsi les bouffées. La largeur de fente du hacheur est réglable. Le faisceau passe ensuite dans le collimateur sous vide de longueur $D_{c}=4 m$ dont les fentes $f_{e}$ (entrée) et $f_{s}$ (sortie), réglables elles aussi, permettent de fixer la résolution angulaire du faisceau (voir ci-dessous). Louverture des fentes peut varier, sur le réflectomètre EROS, de 0,5 à $4 \mathrm{~mm}$. Le faisceau, horizontal, se réfléchit sur l'échantillon solide incliné d'un angle $\theta$ par rapport au faisceau. Langle de mesure maximum est de $5^{\circ}$ et la gamme de q disponible est de 0,004 à $0,1 \AA^{-1}$. (Remarquons qu'une courbe de réflectivité est souvent acquise en plusieurs mesures, l'angle $\theta$ variant d'une mesure à lautre. Le plus souvent une première acquisition à $\theta=1^{\circ}$ permet d'obtenir le plateau de réflexion totale et les points aux grandes valeurs de la réflectivité, puis une seconde mesure avec un angle de l'ordre de $3^{\circ}$ à $5^{\circ}$ permet de mesurer la courbe aux grands q.) Le faisceau réfléchi arrive enfin sur le détecteur à ${ }^{3} \mathrm{He}$ où sont comptés les neutrons en fonction de leur longueur d'onde. La réflectivité minimum que l'on peut atteindre est de l'ordre de $10^{-5}$. Le détecteur est muni d'un mouvement permettant de changer sa position verticale. Les positions $h_{0}$ et $h_{d} d u$ détecteur sont telles que les faisceaux direct et réfléchi respectivement rencontrent le détecteur. L'angle $\theta$ de mesure désiré détermine la hauteur du détecteur selon :

$$
\tan (2 \theta)=\frac{h_{d}-h_{0}}{D_{\text {ech }- \text { dét }}}
$$

où $\mathrm{D}_{\text {éch-dét }}$ est la distance entre l'échantillon et le détecteur.

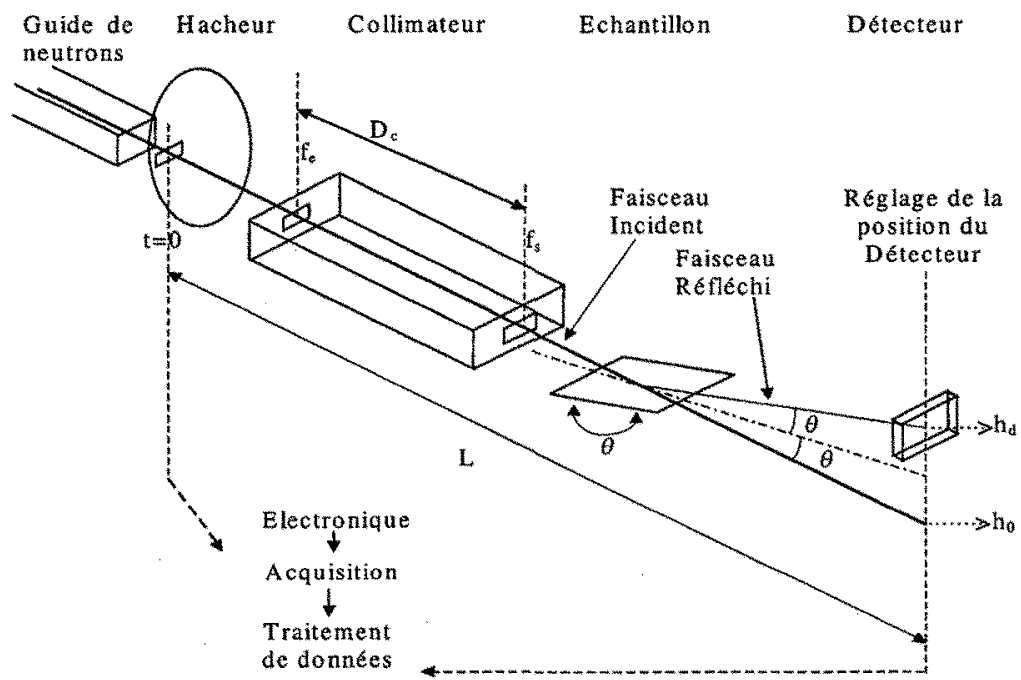

Figure 7: Schéma de principe du réflectomètre EROS. 


\section{Résolution angulaire}

La divergence du faisceau incident $\delta \theta$ ainsi que la résolution angulaire $\delta \theta / \theta$ sont déterminées par l'ouverture des fentes d'entrée et de sortie du collimateur ainsi que par la longueur du collimateur $\mathrm{D}_{\mathfrak{c}}$. Si l'on néglige l'effet de la gravité terrestre, la trajectoire du faisceau peut être supposée rectiligne (voir Figure), et alors la divergence est donnée par :

$$
\tan \delta \theta=\frac{f_{e}+f_{s}}{2 D_{c}}
$$

Le calcul de la divergence et de la résolution pour un angle de $1,5^{\circ}$, des fentes $\mathrm{f}_{\mathrm{e}}=\mathrm{f}_{\mathrm{s}}=\mathrm{lmm}$ et pour $\mathrm{D}_{\mathrm{c}}=3890 \mathrm{~mm}$ donne : $\delta \theta=0,015^{\circ}$ et $\delta \theta / \theta=1 \%$.

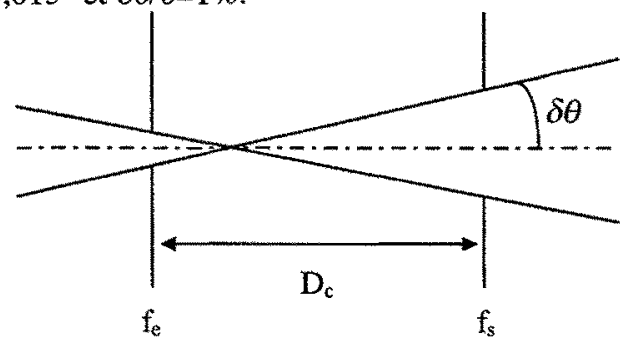

Figure 8: Divergence du faisceau incident lorsque l'on néglige l'effet de la gravité terrestre sur la trajectoire des neutrons.

\section{CRISP}

Le réflectomètre CRISP ${ }^{6}$ est construit sur le même principe que le réflectomètre EROS. La source de neutrons est cependant différente, il s'agit ici de la source pulsée ISIS. ${ }^{7}$ La gamme de longueur d'onde disponible est de 1,5 à $13 \AA$, l'angle incident maximum est de $3^{\circ}$ environ pour les solides. La gamme maximale en $Q$ utilisable sur ce réflectomètre est de 0,01 à $1,3 \AA^{-1}$. Cependant, on peut travailler avec des longueurs d'onde allant de 1,5 à $6,5 \AA$ afin de disposer d'un flux plus important, et à $\theta=2,6^{\circ}$. La gamme en $\mathrm{Q}$ dont on dispose devient alors de 0,1 à $0,4 \AA^{-1}$. La détection du faisceau est assurée par un compteur simple ou un multidétecteur linéaire. La réflectivité minimale détectable est de $10^{-6}$. (la gamme $[1,5 ; 13] \AA$ n'est accessible que grâce à un chopper qui, en éliminant une bouffée sur deux (d'où une diminution du flux), permet le non recouvrement des neutrons les plus lents de la bouffée $n$ avec les plus rapides de la bouffée $n+1$ ).

L'acquisition de données avec ce réflectomètre permet de compléter les mesures faites sur le réflectomètre EROS jusqu'à $q=0,2 \AA^{-1}$ et/ou $R=10^{-6}$. L'intérêt est qu'un nombre maximum d'informations sur l'échantillon permet de le caractériser avec le maximum de précision. En particulier, la courbe aux grandes valeurs de $q$ est indispensable pour déterminer les rugosités d'interfaces. La Figure 9 illustre la complémentarité des mesures. 


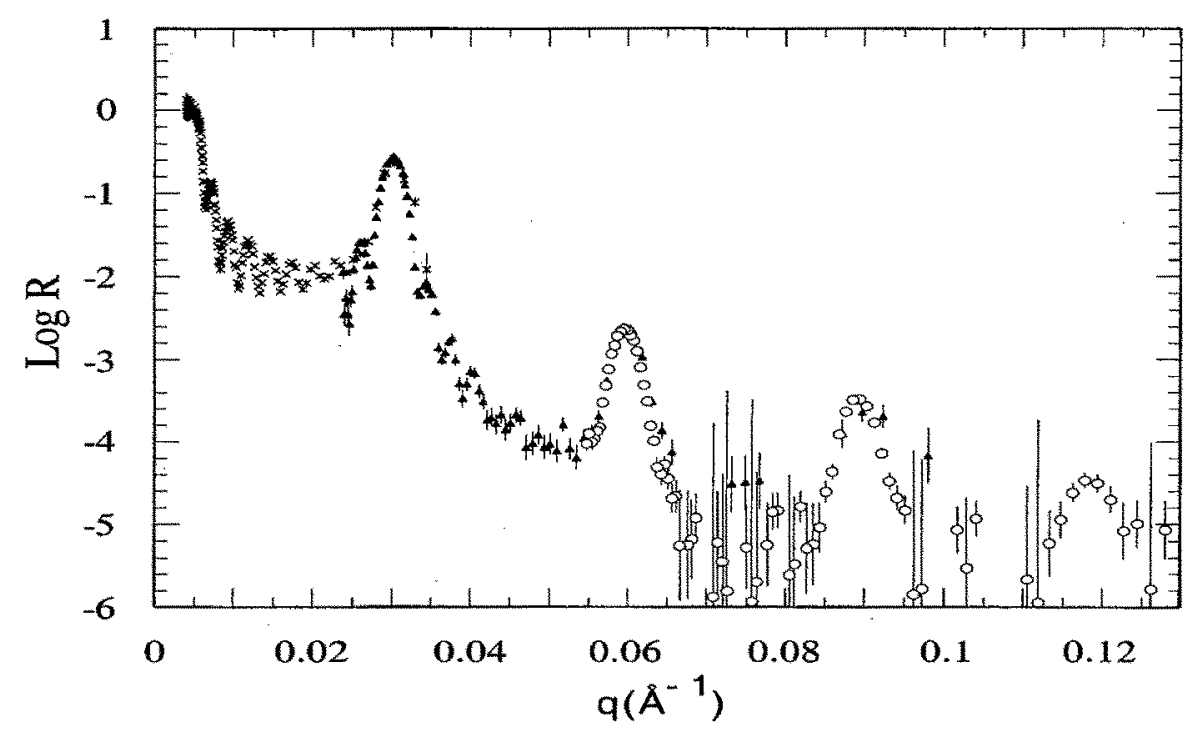

Figure 9 : Exemple de courbe de réflectivité de neutrons acquise en plusieurs mesures sur les deux réflectomètres EROS et CRISP:

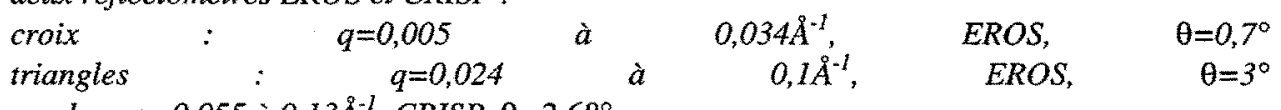

cercles : $q=0,055$ à $0,13 \AA^{-1}$, CRISP, $\theta=2,68^{\circ}$

PRISM

PRISM est un réflectomètre en neutrons polarisés 2 axes $\theta-2 \theta$ (langle entre le faisceau direct et l'échantillon est égal à $\theta$, celui entre le faisceau direct et le détecteur est égal à $2 \theta$ ). De même que pour le réflectomètre EROS, les neutrons sont produits par le réacteur Orphée puis conduits par un guide neutrons jusqu'au spectromètre. Le faisceau polychromatique du guide est filtré par un monochromateur à $\lambda=4,2 \AA$ et dévié à l'extérieur du guide. Le faisceau monochromatique ainsi obtenu est polarisé par un miroir polarisant (seuls les neutrons dont les spins sont orientés dans le même sens sont conservés). Le faisceau est réfléchi sur l'échantillon dont l'orientation $\theta$ par rapport au faisceau varie au cours de la mesure. L'angle incident maximum est de $70^{\circ}$. Un analyseur permet ensuite de trier les neutrons selon lorientation de leur spin. Lintensité du faisceau réfléchi est mesurée en fonction de l'angle grâce à un détecteur simple à ${ }^{3} \mathrm{He}$.

Une description du réflectomètre et les programmes de simulation associés peuvent être trouvé sur le site du laboratoire léon Brillouin :www-llb.cea.fr 


\subsection{Réflectivité de rayons $\mathrm{X}$}

\section{Principes}

La réflectivité de rayons $X$ et la réflectivité de neutrons sont basées sur les mêmes principes. La différence entre les deux techniques se situant au niveau des indices de réfraction. ${ }^{8}$ Les rayons $\mathrm{X}$ sont sensibles à la densité électronique des atomes. Contrairement aux neutrons, l'indice de réfraction en rayons $\mathrm{X}$ est fonction du numéro atomique $\mathrm{Z}$ et la composante d'absorption n'est pas négligeable. Il est défini par :

$$
\begin{aligned}
& n=1-\delta+i \beta \\
& \delta=\frac{r_{0} \lambda^{2}}{2 \pi} \rho\left(Z+\Delta f^{\prime}\right) \\
& \beta=\frac{r_{0} \lambda^{2}}{2 \pi} \rho \Delta f^{\prime \prime}=\frac{\lambda}{4 \pi} \mu
\end{aligned}
$$

où $\mathrm{r}_{0}$ est le rayon classique de l'électron $\left(\mathrm{r}_{0}=2,82 \times 10^{-15} \mathrm{~m}\right), \lambda$ la longueur des rayons $\mathrm{X}$ incidents, $\mathrm{N}$ le nombre d'atomes par unité de volume, $Z$ le numéro atomique, $Z+\Delta f^{\prime}$ et $\Delta f$ " sont les parties réelles et imaginaires du facteur de diffusion et $\mu$ le coefficient d'absorption. Les tables de Henke répertorient les valeurs de f', $\mathrm{f}$ " et $\mu$ en fonction de la longueur d'onde incidente. L'indice n peut s'écrire sous la forme :

$$
n_{X}=1-\frac{\lambda^{2}}{2 \pi} \rho r_{0}\left(Z+f^{\prime}+i f^{\prime \prime}\right)
$$

qui est analogue à l'expression de n pour les neutrons : $n=1-\frac{\lambda^{2}}{2 \pi} \rho b$ (voir chapitre I).

Les réflectomètres utilisés pour nos mesures fonctionnent à longueur d'onde constante $\left(\mathrm{K}_{\mathrm{a}} \mathrm{Cu}=1,54 \AA\right)$ et font varier l'angle incident. Cette configuration permet d'atteindre facilement des valeurs élevées de $\mathrm{q}$.

\section{Intérêt de l'utilisation de cette technique}

La réflectivité de rayons $X$ est complémentaire de la réflectivité de neutrons, et est souvent utilisée afin de diminuer le nombre de paramètres inconnus lors de l'ajustement numérique des courbes de réflectivité de neutrons. Pour cela, on utilise les caractéristiques suivantes de la réflectivité $\mathrm{X}$ :

- Les courbes de réflectivité $X$ sont dépendantes des mêmes paramètres de dépôts que les courbes de neutrons. Outre l'indice de réfraction dont la valeur numérique varie entre les $X$ et les neutrons, les valeurs des épaisseurs et des rugosités se retrouvent d'une technique à l'autre ;

- Le flux incident est plus important, permettant ainsi d'atteindre des faibles valeurs de la réflectivité ;

- La gamme en q est plus importante. 
On a vu, que la position des franges de Kiessig (pour un échantillon simple couche) ou des pics de Bragg (pour un échantillon monochromateur) est fonction de l'indice $\rho$ b aux faibles valeurs de $q$. La gamme en q étant limitée sur les courbes de réflectivité de neutrons, la détermination des épaisseurs à partir de quelques franges seulement augmente la valeur de l'incertitude. Ceci est particulièrement vrai pour les échantillons mesurés sur une faible gamme de $\mathrm{q}$.

La mesure d'un même échantillon par réflectivité $X$ et de neutrons permet de déterminer la valeur de son épaisseur (ou de sa période) avec une plus grande précision en faisant interagir les deux résultats :

Une première valeur de l'épaisseur est déterminée à partir de la courbe X. Cette valeur est d'autant plus précise que le nombre de franges (ou de pics) est grand. Elle est ensuite injectée dans le jeu de paramètres de la courbe de neutrons, puis modifiée au cours de l'ajustement de cette courbe.

Cette méthode permet non seulement de diminuer le nombre de paramètres inconnus lors du traitement de données neutrons, mais encore de découpler l'effet du $\rho b$ et de l'épaisseur aux faibles valeurs de $\mathrm{q}$, et donc de déterminer les valeurs de ces deux paramètres avec une plus grande précision.

Par ailleurs, on peut également traiter les mesures de réflectivité $\mathrm{X}$ avec les mêmes programmes et algorithmes d'ajustement que les mesures de réflectivité neutrons, seul les indices et les absorption étant différents.

\section{Exemples de polymères}

\subsection{Interdiffusion entre deux couches}

La réflectivité est en général bien adapté aux mesures sur les systèmes polymèriques car les distances mesurées, de l'ordre de $100 \AA$, sont du même ordre de grandeur que les tailles des polymères. De plus, du fait de la grande différence de longueur de diffusion entre l'hydrogène $\left(b=-0.374110^{-12} \mathrm{~cm}\right)$ et le deutérium $\left(b=0.667410^{-12} \mathrm{~cm}\right)$, on obtient par deutération sélective de l'un des constituants un excellent contraste pour les mesures de réflectivité de neutrons. L'utilisation de la technique dite de 'spin coating' permet la préparation de film mince de polymère déposés sur verre ou silicium ayant des rugosités de surface de l'ordre de $10 \AA$. Par flottage, on peut ensuite venir déposer une seconde couche sur la première couche. On peut ainsi étudier la diffusion des polymères entre les deux couches en fonction des températures de recuit, et des temps de recuit. Cette diffusion se traduira par un élargissement de l'interface entre les deux couches. Sur les courbes de réflectivité, la signature de l'élargissement sera un amortissement des franges de Kiessig ainsi que représenté sur la figure suivante. 


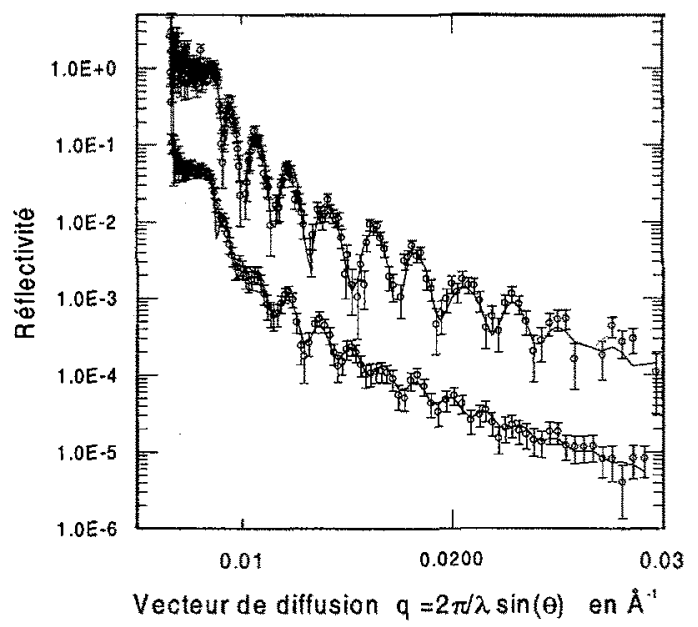

Figure 10: Réflectivité mesurée sur un échantillon bicouches: une couche de polystyrène non deutérié a été déposé sur une couche de polystyrène deutérié. La courbe inférieure correspond à la mesure du même échantillon après un recuit de lheure à $115^{\circ} \mathrm{C}$.

(Pour des raisons de clarté, la courbe inférieure a été décalée vers le bas d'un facteur 0.05)

\subsection{Interdiffusion entre plusieurs couches}

Les copolymères biséquensés symétriques sont composés de deux chaînes $\mathrm{A}$ et $\mathrm{B}$ de longueur identique soudées bout à bout. Ces sytèmes présentent un grand nombres de propriétés intéressantes. Par exemple si A et B sont immiscibles, ils ont la faculté de s'organiser en couches d'épaisseurs fixes parrallèles à la surface lorsqu'ils sont déposés par la technique dite du 'spin coating' puis recuit. L'utilisation de polymères marqués (deutérés) et non marqués permet d'étudier par réflectivité de neutron la diffusion des molécules perpendiculairement à la surface. Les résultats d'une telle mesure sont présentés sur les figures suivantes.
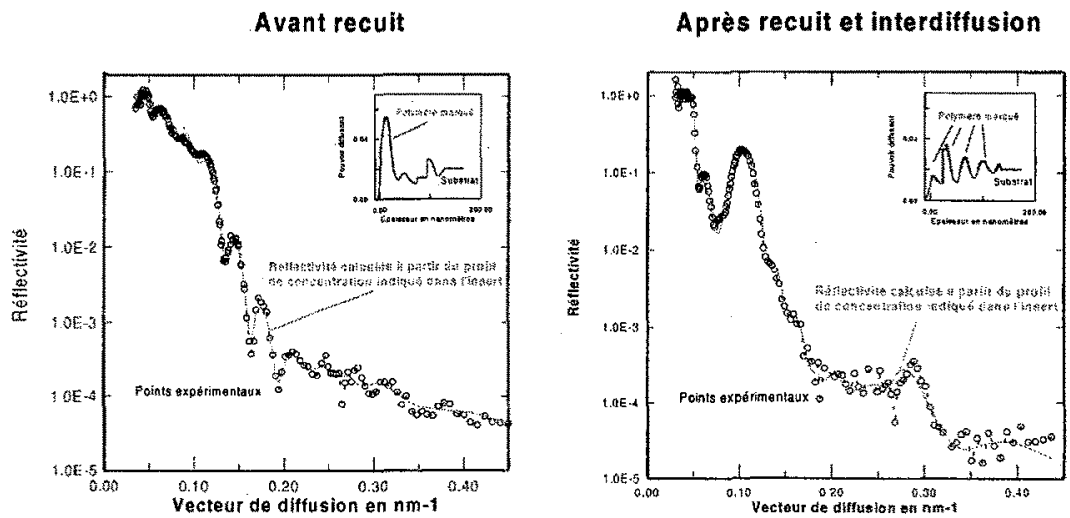

Figure 11: Le sytème initial est représenté sur la figure de gauche. Il s'agit d'une couche de polymère biséquensé marquée déposée sur une tricouche couche de polymère biséquensé non marqué. La courbe de réflectivité présente seulement quelques franges de Kiessig. 
Le système a ensuite été recuit pendant $12 \mathrm{~h} \mathrm{à} 120^{\circ} \mathrm{C}$, puis mesuré une nouvelle fois (courbe de droite). Cette fois on voit clairement apparaître un pic de Bragg. Il correspond à une diffusion du polymère marqué de la couche de surface vers les couches inférieures.

\section{Exemples magnétiques}

Dans cette partie, je donne quelques exemples qui mettent en évidence les différentes informations que l'on obtient sur des couches minces. L'ensemble des résultats expérimentaux montrés ont été réalisés sur PRISM.

\subsection{Mesure absolue de moments magnétiques}

Dans le cas de couches simples ou d'alliages binaires, il est possible de mesurer très précisément le moment magnétique absolu (en $\mu_{B}$ par atome). C'est la seule technique qui permet cela. Les mesures d'aimantation donnent une valeur dépendant du volume et le dichroïsme magnétique donne un moment moyennant une connaissance du nombre d'occupation. On peut donc se servir des neutrons comme calibration d'autres techniques.

A titre d'exemple, la figure 12 donne la réflectivité d'un alliage de NiFe. En ajustant les courbes, on obtient la concentration en $\mathrm{Ni}$ et en $\mathrm{Fe}$ à $1 \%$ près ainsi que le moment de l'alliage avec une précision de $0.02 \mu_{\mathrm{B}}$ environ. Cette mesure a pris 15 minutes sur un échantillon d'un $\mathrm{cm}^{2}$.

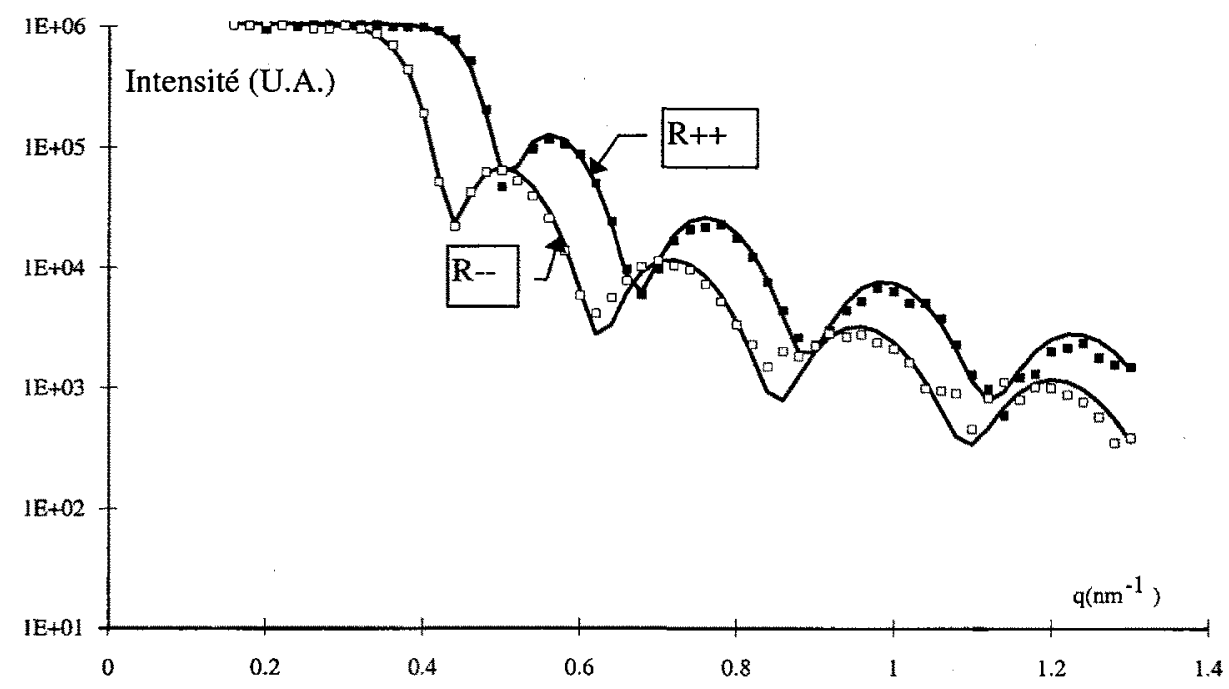

Figure 12: courbe de réflectivité d'une couche de $25 \mathrm{~nm}$ de $\mathrm{NiFe}$. 


\subsection{Pics de Bragg magnétiques d'une multicouche et supermiroirs}

\subsubsection{Multicouches}

Dans le cas d'une multicouche, on peut observer aux petits angles les pics de Bragg correspondant aux périodes de celles-ci. Il est ainsi possible de caractériser rapidement le caractère antiferromagnétique ou ferromagnétique du couplage entre couches. Dans le cas de couches antiferromagnétiques on peut mesurer l'aimantation absolue de chaque couche.

Dans l'exemple ci-dessous, je montre une multicouche Cobalt-titane. La particularité de cette couche est la valeur négative de b dans le cas d'un état de spin. Il s'ensuit une très forte polarisation sur le pic de Bragg. C'est en se servant de telles multicouches que l'on peut polariser les neutrons.

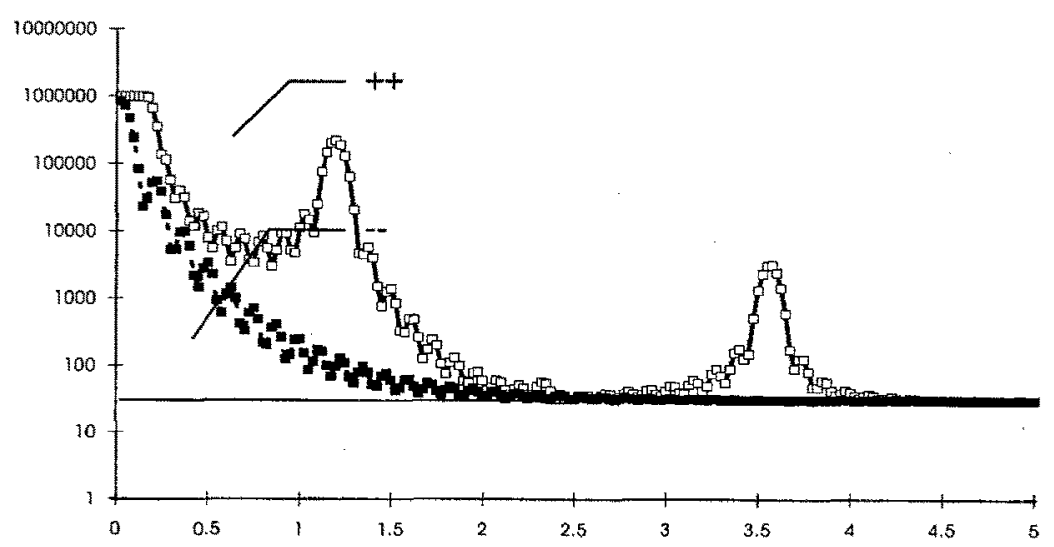

Figure $13: 30$ couches de Co/Ti $(50 \AA, 50 \AA)$ polarisées le long du champ (simulation).

\subsubsection{Principe du supermiroir}

Supposons que l'on construise une multicouche composée d'une variation continue d'épaisseur. Cela revient à placer de nombreux pics de Bragg à partir du plateau de réflectivité totale naturelle. On peut ainsi artificiellement augmenter la taille du plateau de réflectivité totale d'un facteur 3 ou même 4. Cela induit un gain en flux très important. 


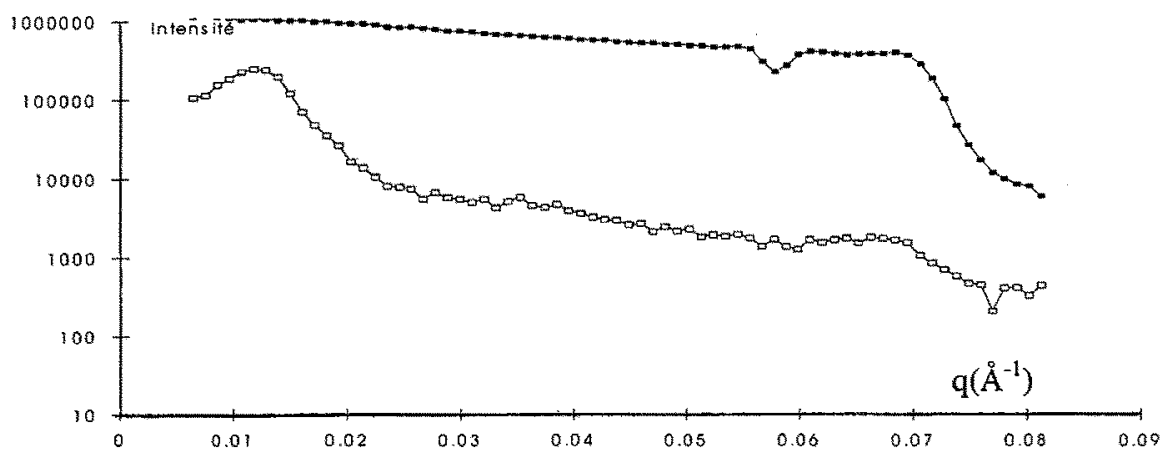

Figure 14 : exemple de polariseur $3 \theta_{c}$ (les deux courbes montrent $R++$ et $R-$ ) Le petit trou dans le plateau artificiel est du a un défaut de la séquence de la multicouche et il montre bien la construction artificielle de ce plateau.

De même que l'on construit un supermiroir à partir d'une série de pics de diffraction de Bragg, on peut construire un supermiroir polarisant à base de $\mathrm{Co} / \mathrm{Ti}$. On fabrique ainsi des objets ayant une acceptance angulaire ou en longueur d'onde raisonnable. Le petit défaut à $0.06 \AA^{-1}$ vient d'un trou dans la variation de période.

\subsection{Voir des tout petits moments}

Supposons que l'on veuille mesurer l'aimantation d'une monocouche d'un métal magnétique.

Si cette couche est placée à la surface de l'échantillon, on ne pourra pas mesurer son aimantation. Par contre, placée entre deux autres couches, le piégeage du neutron dans ce puits de potentiel sera visible sur la réflectivité et il sera tout à fait possible de mesurer le moment correspondant.

Je donne ici un exemple théorique d'une monocouche de fer saturé coincée entre deux matériaux non magnétiques (exemple $W$ ). Cette expérience a été en autre réalisée sur le réflectomètre polarisé de Dubna par une équipe du Laboratoire Louis Néel [15].

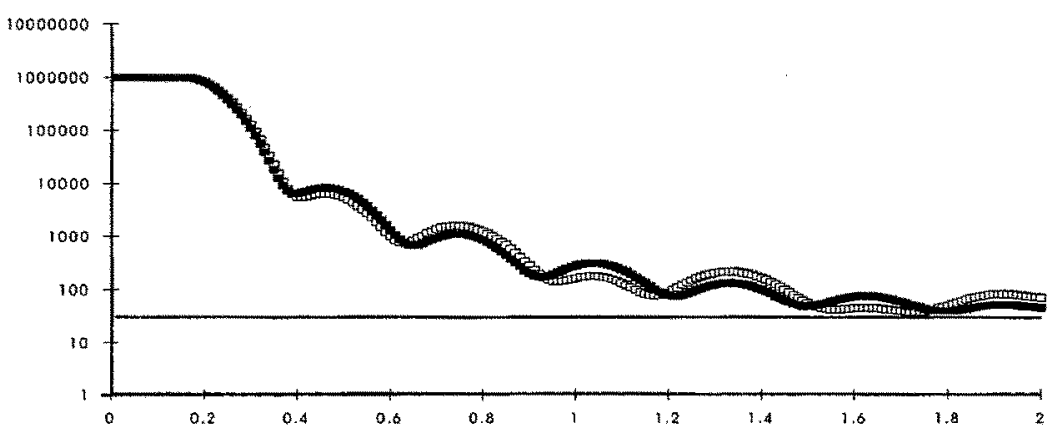

Figure 15 : Courbe pour l'aimantation parallèle au champ extérieur pour une tricouche $W / F e(2 A) / W$ 


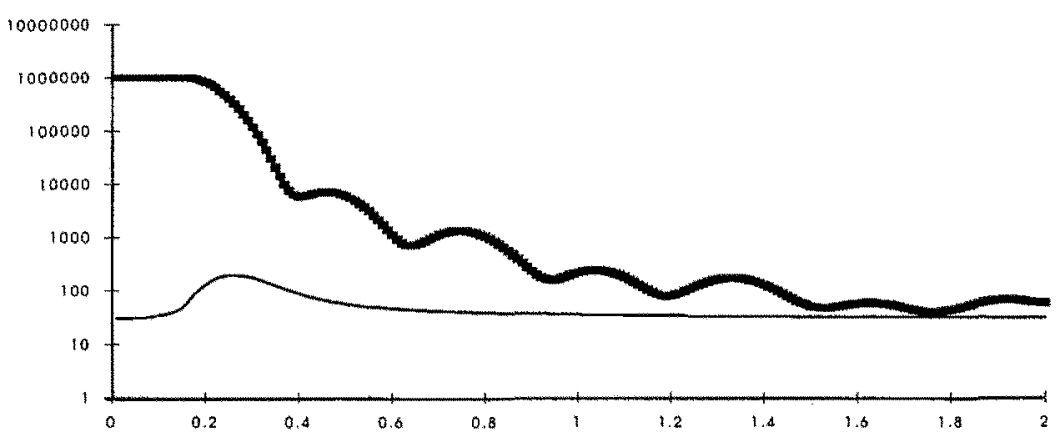

Figure 16: Courbe pour l'aimantation perpendiculaire au champ extérieur pour une tricouche $W / F e(2 \AA) / W$

Voir un signal à partir de rien semble à priori plus efficace, en pratique le signal de spin flip est très petit dans le cas de la figure 16 et sera noyé dans le défaut des flippers et des polariseurs. On aura donc fortement intérêt à travailler dans le cas de la figure 15 .

Une grandeur très intéressante est le rapport de flipping entre les courbes (++) et (-)
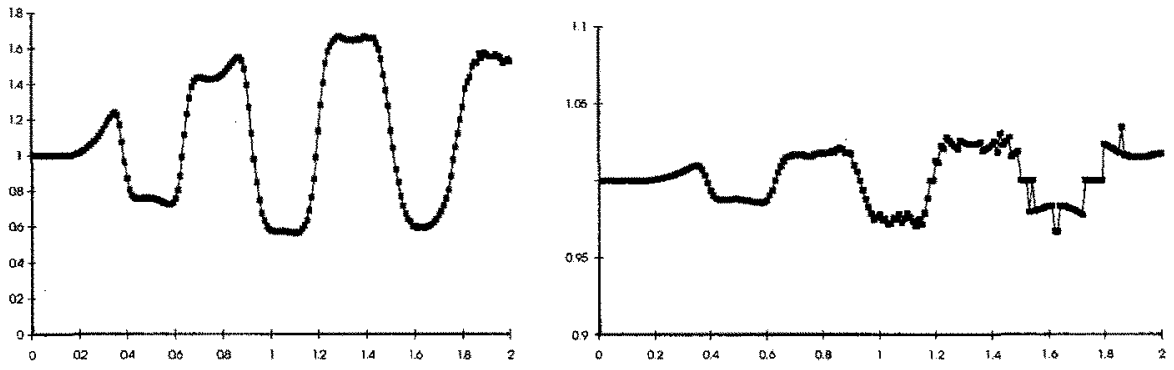

Figure 17 : Rapport de flipping pour une couche W/Fe $(2 \hat{A}) / W$ avec $2.2 \mu_{B}$ et $0.1 \mu_{B}$

Une seule couche atomique avec un moment de $0.1 \mu_{\mathrm{B}}$ est facilement mesurable grâce à l'apport de l'analyse de polarisation.

On voit de plus ici, un exemple intéressant de la brisure de la loi en $2 \pi / \mathrm{q}_{\max }$. On s'arrête à la mesure d'une taille à priori de l'ordre de $25 \AA$, en raison de la faible extension en angle de la mesure et pourtant on est sensible à une épaisseur beaucoup plus fine. C'est ce type d'effet qui empêche l'application de procédures d'inversion de la courbe de réflectivité.

\subsection{Tracer des cycles d'hysteresis 1d ou 2d}

Lors d'une étude en champ ou température, on ne peut pas réaliser un grand nombre de courbes de réflectivité.

Le principe consiste donc à faire une courbe en champ saturant l'analyser, en tirer les différents paramètres (épaisseurs, densités, moments) puis à ne mesurer que $\mathrm{N}+1$ points où $\mathrm{N}$ est le nombre d'inconnues (moments des couches par exemple). On ajuste en fin ces $\mathrm{N}$ paramètres, les autres étant connus.

Ainsi une mesure à un champ donné ne prend que très peu de temps. 


\subsubsection{Cas d'une simple couche magnétique}

$\mathrm{Au} / \mathrm{Co}(50 \mathrm{~nm}) / \mathrm{Au}$ tri-layer.
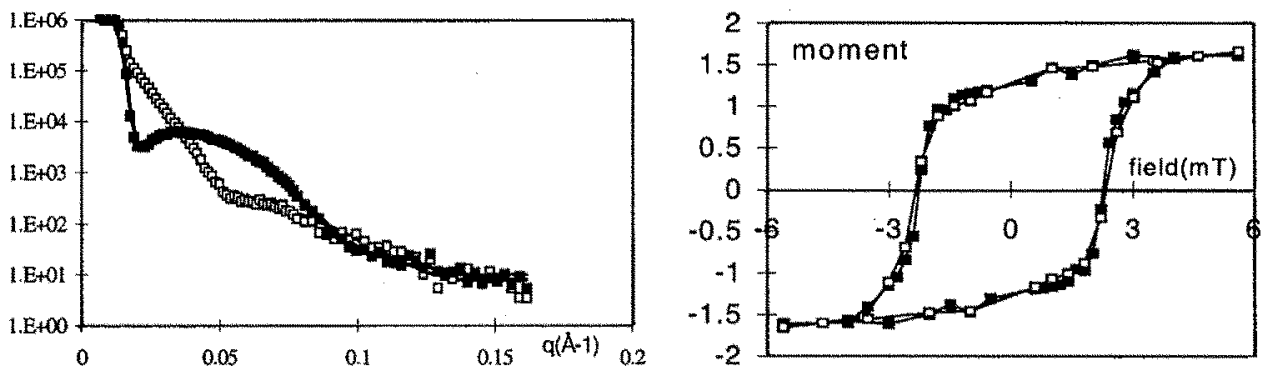

figure 18: courbe de réflectivité d'une couche de 50nm de cobalt et cycle d'hystéresis déterminé à 0.3 et $0.6^{\circ}$

\subsection{2 deux couches magnétiques}

Substrat $/ \operatorname{Pt}(98 \AA) / \operatorname{Co}(7.6 \AA) / \operatorname{Pt}(33 \AA) / \operatorname{Co}(3.8 \AA) / \operatorname{Pt}(32 \AA)$.
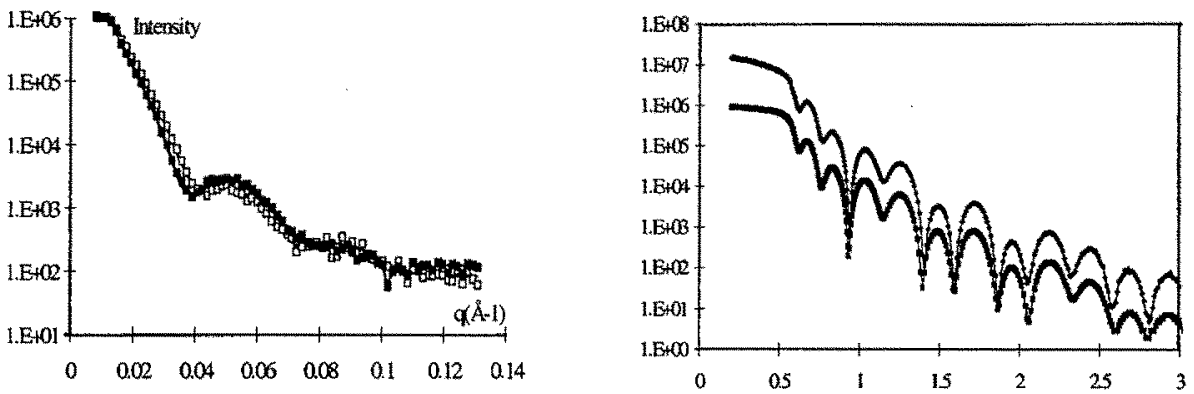

Figure 19 : courbes de réflectivité neutrons et $X$

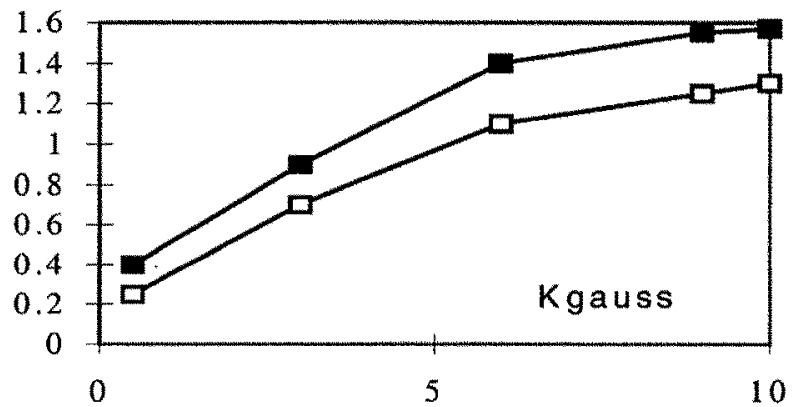

Figure $20:$ moments déterminés par ajustement sur 3 points. Les points noirs correspondent à la couche de $7.6 \AA$ et les points blancs à la couche de $3.8 \AA$. 


\subsection{Mesure de la rotation de moments dans le plan ou hors du plan}

C'est dans ce domaine que la réflectivité neutronique avec analyse de polarisation a un apport essentiel. C'est l'information la plus fine et la plus intéressante que l'on obtient par cette technique. Je me contente de donner un exemple ici sur un tout petit échantillon

\subsubsection{Mesure de l'aimantation de surface d'un whisker de fer}

Faire un monocristal de fer est extrêmement difficile. Actuellement très peu de gens savent réaliser de tels échantillons. Nous avons étudié un tel monocristal qui présente une face incroyablement plane (1 marche atomique tous les microns) fabriqué par l'équipe de B. Heinrich à Vancouver. La difficulté est la taille de l'échantillon $: 7 \mathrm{~mm}^{2}$. Nous avons tout de même réussi à mesurer l'aimantation de surface en direction et en amplitude.

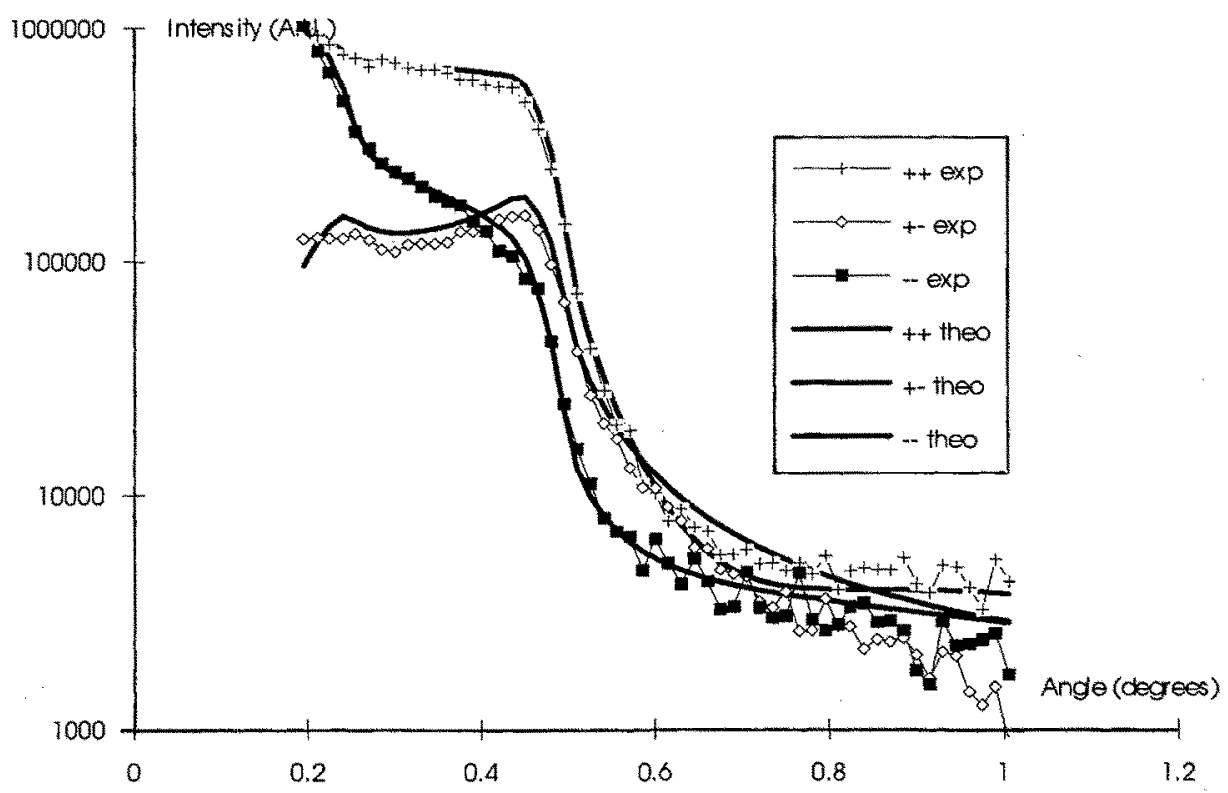

Figure 21: réflexion sur un monocristal de fer (surface $7 \mathrm{~mm}^{2}$ ) en analyse de polarisation.

Le moment trouvé est de $2.21 \mu_{\mathrm{B}} \pm 0.01 \mu_{\mathrm{B}}$ et un angle à la surface de $28^{\circ}$ à environ $45^{\circ}$ de la direction angulaire dans le bulk. Nous n'avons pas d'explications à cela excepté un défaut de croissance situé à environ $250 \mathrm{~nm}$ de la surface. Cette expérience a permis de montrer la possibilité d'étudier de tout petits échantillons. 


\section{Conclusion sur la réflectivité de neutrons}

J'espère avoir montré la richesse que peut apporter la réflectivité de neutrons polarisés au magnétisme des couches minces. Il y aurait encore beaucoup de choses à dire.

Un autre aspect non abordé est l'aspect inélastique de la réflectivité. On peut en effet montrer que la direction du faisceau réfléchi est directement liê à la perte ou au gain d'énergie lors du processus. On aurait ainsi la possibilité de mesurer des excitations de surface près de $q=0$. La seule mesure de gain ou perte d'énergie du neutron jusqu'à maintenant réalisée est simplement la différence d'énergie Zeeman lors d'une réflexion avec spin-flip. On retrouve alors des équations (11). que les faisceaux $\mathrm{R}+$ - et $\mathrm{R}+$ sont de part et d'autre de $\mathrm{R}++$ et $\mathrm{R}--$, La différence angulaire est directement liée au champ extérieur appliqué. Cela a été récemment mesuré par G. Felcher [14].

\section{Références}

1. . J.C. Charmet, P.G. De Gennes, J. Opt. Soc. Am., 73, 12, pp.1777-84, 1983

2. A. Penninckx-Sans, Communication Privée

3. .E. Fermi, Nuclear Physics, University of Chicago Press, 1949

4. . B. Farnoux, Neutron Scattering in the 'nineties, Conf. Proc. IAEA in Jülich, 14-18 janvier 1985, pp. 205-209, Vienne, 1985

5. . X.D. Sun, Thèse de l'Université Paris Sud, Orsay, 1988.

6. . J. Penfold, R.K. Thomas, J. Phys. Condens. Matter, 2, pp.1369-1412, 1990

7. . J. Penfold, R.C. Ward, W.G. Williams, J. Phys. E. : Sci. Instrum., 20, p.1411, 1987

8. . T.P. Russel, Mat. Sc. Rep. 5, pp.171-271, 1990

9. Erwin and al, Phys Rev. B 35 (1987) p 6808.

10.Methods of experimental physics, volume 23 part $C$

11.C. F. Majkrzak, J. W. Cable, J. Kwo, M. Hong, D. B. McWhan, Y. Yafet and J. Waszcak, Phys. Rev. Lett. 562700 (1986).G.P. Felcher, R.O. Hilleke, R.K. Crawford, J. Haumann, R. Kleb and G. Ostrowski, Rev. Sci. Instr 58, 609 (1987).

12.S.J. Blundell and J.A.C. Bland, Phys. Rev.B 46, 3391 (1992)

13.Pleshanov Z. Phys B 94(1994) p 233-243

14.felcher nature aout 1996

15.O. F. K. McGrath, thèse de l'université Joseph Fourier, Grenoble 1994.

Il faut rajouter :

Leckner : «theory of réflexion »(Dordecht :Martinus Nijhoff) (1987). 